\title{
Spatially Modulated Code-Division Multiple-Access for High-Connectivity Multiple-Access
}

\author{
Peng Pan, Member, IEEE, and Lie-Liang Yang, Fellow, IEEE
}

\begin{abstract}
In order to take the advantages of spatial modulation (SM) and support the multiple access (MA) communications requiring high-connectivity, we integrate the SM with DS-CDMA to propose a SM/DS-CDMA scheme, which is in favor of attaining both antenna diversity and frequency diversity. Associated with the SM/DS-CDMA scheme, a range of linear and nonlinear multiuser detectors (MUDs) are proposed and studied. Specifically, for linear MUDs, both minimum mean-square error aided joint spatial demodulation (MMSE-JSD) and MMSE-aided separate spatial demodulation (MMSE-SSD) are introduced. For nonlinear MUDs, we first present the joint maximum-likelihood MUD (JML-MUD) for demonstrating the potential of SM/DSCDMA systems. Then, the MMSE-relied iterative interference cancellation (MMSE-IIC) is suggested, and associated with which two types of low-complexity high-efficiency reliability measurement schemes are proposed. In this paper, we mathematically analyze both the single-user average bit error rate (ABER) of the SM/DS-CDMA systems employing joint spatial demodulation (JSD), and the approximate ABER of the SM/DS-CDMA systems employing the MMSE-JSD. We investigate and compare the ABER performance of the SM/DS-CDMA systems with various detection schemes, and also with some legacy schemes. Our studies and performance results show that the SM/DS-CDMA systems in conjunction with appropriate MUD schemes are capable of providing a desirable trade-off among the number of users supportable, implementation complexity and the ABER performance.
\end{abstract}

Index Terms-MIMO, spatial modulation, CDMA, space shift keying, multiuser detection, interference cancellation, performance analysis.

\section{INTRODUCTION}

Spatial modulation (SM) [1-3], which conveys information using jointly space shift keying (SSK) and conventional amplitude-phase modulation (APM), has drawn a lot of attention in research in recent years, and has been investigated from different perspectives, as shown by the overview, e.g., in [4-6]. In the domain of SM, recent studies include lowcomplexity transceiver design [7-11], error performance and capacity analysis [12-16], full-duplex SM [17-19], compressive sensing assisted detection [20], channel estimation [21, 22], physical-layer security [18, 23-25], massive MIMO based SM [26-28], etc.

In this paper, our focus is on the multiple-access (MA) communications providing high-connectivity in massive device (machine-type) communications environments [29]. On the topic of MA, in [30], a two-user SM system with either single-user or joint maximum likelihood multiuser detection

P. Pan is with the School of Communications Engineering, Hangzhou Dianzi University, Hangzhou, China. (E-mail: panpeng@hdu.edu.cn). L.-L. Yang is with the School of Electronics and Computer Science, University of Southampton, SO17 1BJ, UK. (E-mail: 1ly@ecs.soton.ac.uk, http://wwwmobile.ecs.soton.ac.uk/lly). The work of P. Pan was supported by the Nationa Natural Science Foundations of China under Grant U1709220. The work of L.-L. Yang was supported in part by the EPSRC, UK, under Project $\mathrm{EP} / \mathrm{P} 034284 / 1$ and in part by the Innovate UK project.
(JML-MUD) has been considered. This two-user MA scheme has been extended to a general SM-assisted MA scheme in [31], where both single-user (interference-unaware) detection and JML-MUD (interference-aware) have been studied. In [32], the author has proposed an antenna-hopping scheme in conjunction with space-shift keying (SSK) modulation for achieving MA and transmit diversity. The authors in [13] have analyzed the error probability of a MA system employing SSK modulation, when assuming communications over Rayleigh fading channels. Furthermore, the references of [20, 26-28, 33, 34] have considered the MA systems with SM in the category of massive MIMO. In all the above-mentioned references, MA is supported by the space (S)-domain resources. Hence, all of them belong to the family of space-division multipleaccess (SDMA), which are hence referred to as the SMmodulated SDMA (SM-SDMA) for convenience of following description. However, in high-connectivity device (machinetype) communications, it is required to support a big number of users (devices, machines), which often results in system full-load or even overload. Consequently, a base-station (BS) receiver in the SM-SDMA systems has to employ a very big number of antennas that is on the scale of the number of users timing the constellation size of SSK [32], in order for low-complexity detection to achieve sufficient reliability. In practice, however, it is highly challenging to deploy a communication terminal, even a BS, with a very large number of antennas, especially, when uncorrected antennas are required. This is because there is usually physical space limit, and if there are many uncorrelated antennas, the system has to put heavy burden on channel estimation, which may consume a lot of system resources, including spectrum resource and energy.

Based on the above considerations, it is desirable to integrate SM with other types of MA techniques [35-37]. It is well known that in cellular wireless systems, direct-sequence codedivision multiple access (DS-CDMA) has been adopted as one of the important MA techniques in the 2nd generation (2G), $3 \mathrm{G}$ and $4 \mathrm{G}$ uplink systems ${ }^{1}$. More importantly, towards the $5 \mathrm{G}$, there are various non-orthogonal MA (NOMA) schemes [39], including low-density spreading CDMA [40,41], grant-free MA (GFMA) [42], non-orthogonal coded access [39,43], etc., having been designed on the basis of DS-CDMA, in order to support high-connectivity MA. Therefore, in this paper, we integrate SM with DS-CDMA, forming the so-called SM/DS-CDMA, in order to support the MA communications with the potential to provide high-connectivity. Note that in literature, the integration of multiantenna MIMO with CDMA (MIMO-CDMA) has been widely studied, as evidenced, e.g.,

${ }^{1}$ Note that, the single-carrier frequency-division multiple-access (SCFDMA) for 4G uplink can be explained as a DS-CDMA scheme that uses DFT matrix for spreading [38]. 
by $[44,49,50]$ and the references therein. In the conventional MIMO-CDMA systems, multiuser detector (detection) (MUD) design can benefit from the knowledge about both users' spreading sequences and the channel state information (CSI) of individual users. By contrast, in our considered SM/DSCDMA systems, the indices of transmit antennas are used by $\mathrm{SM}$ for conveying information in the S-domain. Hence, before detection of the S-domain information, the receiver is unable to know which antenna is activated, and hence unable to tell which part of CSI should be used, even though the receiver employs the CSI from all transmit antennas. Consequently, the CSI is unable to be utilized for the multiuser interference (MUI) suppression executed before detection of the S-domain information. Therefore, the MUD design for SM/DS-CDMA systems is very different from that for the conventional multiuser systems. Recently, a SM-assisted sparse CDMA system has been proposed for supporting potentially a big number of devices [41]. Owing to the employment of sparse spreading, the message passing algorithm (MPA) has been introduced to achieve near-optimum performance but with significantly lower detection complexity than the JML-MUD. However, the MPA detection in [41] depends on the regular graphs, which are not easily scalable and often cannot be guaranteed in practical systems, especially, when active users are dynamic.

In this paper, we introduce a SM/DS-CDMA system having the potential to support high-connectivity, and propose the corresponding signaling and detection schemes. We assume random spreading codes for signaling, and propose the lowcomplexity MUDs that are flexible for operation with different number of users. As the results, our proposed SM/DS-CDMA scheme has the following advantages when compared with the legacy SM-SDMA schemes. First, SM/DS-CDMA exploits the degrees of freedom from both the S-domain provided by multiple receive antennas and the time (T)-domain or code (C)-domain relied on random spreading for supporting multiple users, as well as employs low-complexity flexible detection. It is hence robust and scalable for supporting a large number of and dynamic users, such as, in device (machinetype) communications [29]. Second, the transmitter in SM/DSCDMA system is capable of taking the advantages of SM, such as single RF chain and relaxed antenna synchronization, and is hence feasible for implementation. Third, in comparison with the SM-SDMA schemes, as above-mentioned, the number of receive antennas in a SM/DS-CDMA system can be significantly reduced. Hence, it is more practical to deploy uncorrelated antennas. Furthermore, as shown in our forthcoming discourses, MUI suppression in SM/DS-CDMA systems only makes use of the knowledge about the spreading codes assigned to active users, which are fixed during one transmission. Hence, the matrices used in MUI suppression are only required to be updated, when the state of active users changes. By contrast, in SM-SDMA system, the matrices used for MUI suppression have to be updated either when the state of active users changes or when any of the channels varies. Considering that the number of transmit/receive antennas in a SM-SDMA system for supporting high connectivity is very big, the updating rate of the matrices for MUI suppression should be extremely high. These arguments infer that the complexity of the MUD for SM/DS-CDMA can be significantly reduced in comparison with that for SM-SDMA.

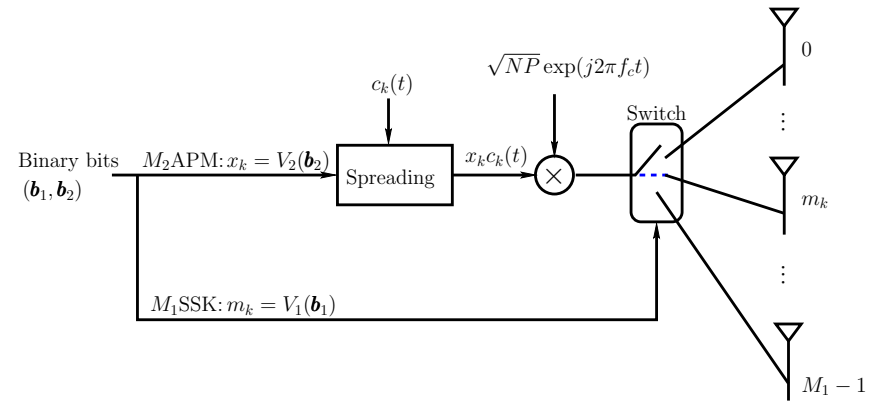

Fig. 1. Transmitter schematic diagram of the $k$ th user in SM/DS-CDMA systems.

In summary, the contributions and novelties of this paper are as follows:

- A SM/DS-CDMA system is proposed and studied with the motivation to support high-connectivity MA in device (machine-type) communications. We assume wideband DS-CDMA relying on random spreading, and experiencing frequency-selective fading. Hence, our SM/DSCDMA system can benefit from the frequency diversity for system performance enhancement.

- Due to the prohibitive complexity of JML-MUD, two classes of low-complexity MUDs are proposed for SM/DS-CDMA. In the first class of MUDs, the linear minimum mean-square error (MMSE) aided MUDs (MMSE-MUDs), which only exploit users' spreading sequences but are independent of CSI, are proposed to suppress MUI. This class of MUDs have very low complexity, but significantly degraded error performance when compared with the JML-MUD. Hence, the second class of MUDs, namely the MMSE-based iterative interference cancellation (MMSE-IIC), are proposed with an acceptable increase of complexity. Furthermore, as the error performance of MMSE-IIC is critically dependent on the reliability measurement of individual users' detection [51], we propose two reliability measurement schemes for MMSE-IIC.

- The average bit error rate (ABER) of the SM/DS-CDMA systems employing representative detection schemes is mathematically analyzed, and corresponding ABER formulas are derived, which allow us to conveniently evaluate the achievable performance of the SM/DS-CDMA systems communicating over frequency-selective fading channels.

The rest of the paper is organized as follows. In Section II, the proposed SM/DS-CDMA system model is described. Then, a range of MUDs for SM/DS-CDMA systems are addressed in Section III. The ABER of some SM/DS-CDMA systems is analyzed in Section IV, and in Section V, some simulation and numerical results are provided to illustrate and compare the achievable ABER performance of SM/DS-CDMA systems. Finally, Section VI summarizes the main conclusions of our research.

\section{System Models}

\section{A. Transmitter}

Let us consider an uplink transmission, where $K$ mobile users synchronously communicate with a BS. We assume that 
BS employs $U$ receive antennas. Although different users may have different numbers of transmit antennas, but in order to avoid dealing with trivial cases, and focus our attention on the main issues concerned, we assume that all users have the same number of transmit antennas, which is expressed as $M_{1}$. Therefore, following the principles of SM and assuming that one of the $M_{1}$ transmit antennas is activated for information delivery $^{2}$, the number of bits conveyed in the S-domain is $b_{1}=\log _{2} M_{1}$. This component symbol is referred to as the $M_{1}$-ary space-shift keying $\left(M_{1} \mathrm{SSK}\right)$ symbol. In addition to the $M_{1}$ SSK symbol, $b_{2}=\log _{2} M_{2}$ bits per symbol is also conveyed via employing a $M_{2}$-ary amplitude phase modulation ( $\left.M_{2} \mathrm{APM}\right)$, such as, $M_{2}$-ary quadrature amplitude modulation (QAM), which is transmitted by the activated transmit antenna. This component symbol is referred to as the $M_{2}$ APM symbol. Consequently, the total number of bits transmitted by a user during a symbol period is $b=\left(b_{1}+b_{2}\right)$ bits. The joint of the $M_{1} \mathrm{SSK}$ and $M_{2} \mathrm{APM}$ symbols is referred to as a SM symbol.

The transmitter schematic diagram of the $k$ th user in a SM/DS-CDMA system is depicted in Fig. 1, which is similar to that in [41], where sparse spreading is employed in order to implement MPA-based detection. As shown in the figure, the $b$-bit symbol is first divided into two component symbols, $\boldsymbol{b}_{1}$ of $b_{1}$ bits and $\boldsymbol{b}_{2}$ of $b_{2}$ bits. The integer value obtained from the mapping of $V_{1}\left(\boldsymbol{b}_{1}\right)$, such as, using natural binary mapping, is expressed as $m_{k}$, which represents the $M_{1}$ SSK symbol. By contrast, the mapping $V_{2}\left(\boldsymbol{b}_{2}\right)$ yields a $M_{2}$ APM symbol expressed as $x_{k}$, which obeys $E\left[\left|x_{k}\right|^{2}\right]=1$. Then, $x_{k}$ is spread using a spreading waveform $c_{k}(t)$. Finally, after the carrier modulation and power amplification, the $k$ th user's signal is transmitted from its $m_{k}$ th antenna determined by the $M_{1}$ SSK symbol $m_{k}$. Correspondingly, the complex baseband equivalent signal transmitted by the $k$ th user can be written as

$$
s_{k}(t)=\sqrt{N P} x_{k} c_{k}(t), \quad k=1,2, \ldots, K
$$

where $N$ is the spreading factor, and $P$ represents the transmitted power. In (1), the DS spreading waveform $c_{k}(t)$ is formed based on the spreading sequence assigned to the $k$ th user, which is expressed as $\boldsymbol{c}_{k}=\left[c_{k 0}, c_{k 1}, \ldots, c_{k(N-1)}\right]^{T}$, and normalized to satisfy $\left\|c_{k}\right\|^{2}=1$. Hence, the spreading waveform can be expressed as $c_{k}(t)=\sum_{n=0}^{N-1} c_{k n} \psi_{T_{c}}\left(t-n T_{c}\right)$, where $\psi_{T_{c}}(t)$ is the chip waveform defined within $\left[0, T_{c}\right)$ and satisfies $T_{c}^{-1} \int_{0}^{T_{c}} \psi_{T_{c}}^{2}(t) d t=1$.

\section{B. Receiver}

Assume that the channel between a user and BS experiences frequency-selective fading, which generates $L$ resolvable paths in the time (T)-domain at the BS receiver. Let us express the discrete-time channel impulse response (CIR) between the $m_{k}$ th transmit antenna of user $k$ and the $u$ th receive antenna at BS as $\boldsymbol{h}_{m_{k}}^{(k, u)}=\left[h_{m_{k}, 0}^{(k, u)}, h_{m_{k}, 1}^{(k, u)}, \ldots, h_{m_{k}, L-1}^{(k, u)}\right]^{T}$, where $h_{m_{k}, l}^{(k, u)}$ is complex Gaussian distributed with zero mean and a variance of $1 /(2 L)$ per dimension. Then, the complex baseband equivalent SM/DS-CDMA signal received by the $u$ th

\footnotetext{
${ }^{2}$ Explicitly, the studies can be readily extended to the generalized SM systems, which simultaneously activate multiple transmit antennas for information delivery in the S-domain.
}

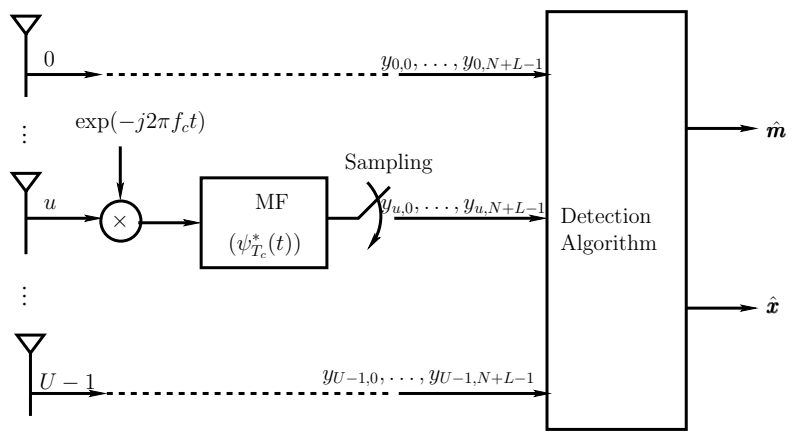

Fig. 2. Receiver schematic diagram of the BS in SM/DS-CDMA systems.

receive antenna of $\mathrm{BS}$ can be expressed as

$$
\begin{aligned}
r_{u}(t)= & \sum_{k=1}^{K} \sum_{l=0}^{L-1} \sqrt{N P} h_{m_{k}, l}^{(k, u)} x_{k} c_{k}\left(t-l T_{c}\right)+n(t) \\
= & \sum_{k=1}^{K} \sum_{n=0}^{N-1} \sum_{l=0}^{L-1} \sqrt{N P} h_{m_{k}, l}^{(k, u)} x_{k} c_{k n} \psi_{T_{c}}\left(t-n T_{c}-l T_{c}\right) \\
& +n(t), u=0,1, \ldots, U-1
\end{aligned}
$$

where $n(t)$ represents the circularly-symmetric complex Gaussian noise process, which has zero mean and a single-sided power-spectrum density (PSD) of $N_{0}$.

The receiver schematic diagram is shown in Fig. 2. Each of the receive antennas employs one matched-filter (MF) matching to the received chip-waveform $\psi_{T_{c}}(t)$, whose output is sampled at the chip rate of $1 / T_{c}$. In this paper, we assume a zero-padding (ZP) DS-CDMA scheme [45-48], where $\mathrm{ZP}$ is applied in order to avoid inter-symbol interference (ISI) [47]. Hence, we can focus our attention on the impact of multiuser interference (MUI). Furthermore, although not implemented in this paper, the ZP relied DS-CDMA schemes are beneficial to employing frequency $(\mathrm{F})$-domain equalization (FDE) techniques $[45,46,48]$. This is because in comparison with the time-domain equalization, whose complexity is proportional to $L$, the complexity of FDE is proportional to $\log L$ [48]. Note that, using ZP introduces some overhead, which is however ignorable, when data are transmitted in blocks or/and when channels are not extremely frequencyselective, resulting in a big value of $L[45,46,49]$. Based on the above settings and assumptions, during a symbol duration, a total $(N+L-1)$ samples can be collected for detection. Specifically, the $(N+L-1)$ observations obtained from the $u$ th receive antenna can be expressed as

$$
\begin{aligned}
\boldsymbol{y}_{u} & =\sum_{k=1}^{K} \boldsymbol{C}_{k} \boldsymbol{h}_{m_{k}}^{(k, u)} x_{k}+\boldsymbol{n}_{u} \\
& =\boldsymbol{C} \boldsymbol{H}_{u}\left(\boldsymbol{m}_{s}\right) \boldsymbol{x}_{s}+\boldsymbol{n}_{u}, u=0,1, \ldots, U-1
\end{aligned}
$$

where $C_{k}$ is a $(N+L-1) \times L$ matrix, each of its columns contains the $k$ th user's spreading sequence $\boldsymbol{c}_{k}$ with its first element $c_{k 0}$ being on the main diagonal of the matrix, while the other elements are zero. The elements of $\boldsymbol{n}_{u}$ are complex Gaussian random variables distributed with zero mean and a variance of $1 /\left(2 \gamma_{s}\right)$ per dimension, where $\gamma_{s}=E_{s} / N_{0}$ denotes the average SNR per symbol. Here, $E_{s}=P N T_{c}$ denotes the energy consumed for sending one SM symbol. In 
the second equation of (3), we have the definitions of

$$
\begin{aligned}
& \boldsymbol{C}=\left[\boldsymbol{C}_{1}, \boldsymbol{C}_{2}, \cdots, \boldsymbol{C}_{K}\right], \\
& \boldsymbol{H}_{u}\left(\boldsymbol{m}_{s}\right)=\operatorname{diag}\left\{\boldsymbol{h}_{m_{1}}^{(1, u)}, \boldsymbol{h}_{m_{2}}^{(2, u)}, \cdots, \boldsymbol{h}_{m_{K}}^{(K, u)}\right\} \\
& \boldsymbol{m}_{s}=\left[m_{1}, m_{2}, \cdots, m_{K}\right]^{T}, \quad \boldsymbol{x}_{s}=\left[x_{1}, x_{2}, \cdots, x_{K}\right]^{T}
\end{aligned}
$$

Let us define

$$
\begin{aligned}
& \boldsymbol{y}=\left[\boldsymbol{y}_{0}^{T}, \boldsymbol{y}_{1}^{T}, \cdots, \boldsymbol{y}_{U-1}^{T}\right]^{T}, \quad \boldsymbol{n}=\left[\boldsymbol{n}_{0}^{T}, \boldsymbol{n}_{1}^{T}, \cdots, \boldsymbol{n}_{U-1}^{T}\right]^{T} \\
& \boldsymbol{H}\left(\boldsymbol{m}_{s}\right)=\left[\boldsymbol{H}_{0}^{T}\left(\boldsymbol{m}_{s}\right), \boldsymbol{H}_{1}^{T}\left(\boldsymbol{m}_{s}\right), \cdots, \boldsymbol{H}_{U-1}^{T}\left(\boldsymbol{m}_{s}\right)\right]^{T}
\end{aligned}
$$

Then, we can represent the $U(N+L-1)$-length vector $\boldsymbol{y}$ as

$$
\boldsymbol{y}=\left(\boldsymbol{I}_{U} \otimes \boldsymbol{C}\right) \boldsymbol{H}\left(\boldsymbol{m}_{s}\right) \boldsymbol{x}_{s}+\boldsymbol{n}
$$

where $I_{U}$ is a $(U \times U)$ identity matrix, and $\otimes$ is the Kronecker production [52].

From above description, we are inferred that multiple users in SM/DS-CDMA system are jointly supported by the Sdomain and C-domain resources, as explicitly shown by the term of $\left(\boldsymbol{I}_{U} \otimes \boldsymbol{C}\right)$ in (6). Hence, in comparison with SMSDMA system [31], the number of receive antennas at a BS of SM/DS-CDMA system can be significantly reduced, when both systems support a similar number of users. Hence, in comparison with SM-SDMA, SM/DS-CDMA is more feasible for BS implementation, as deploying a big number of lowcorrelation antennas is not only limited by BS's physical space, but also adds heavy burden on channel estimation. In Fig. 11, we will compare the BER performance of SM/DS-CDMA with that of SM-SDMA, associated with some further discussion.

\section{Detection Algorithms}

We first consider the optimum JML-MUD, which allows us to gain insight into the potential of SM/DS-CDMA. In order to reduce the detection complexity, we then propose the MMSE aided joint spatial demodulation (MMSE-JSD), MMSE aided separate spatial demodulation (MMSE-SSD), and the MMSEbased iterative interference cancellation (MMSE-IIC).

\section{A. Joint Maximum-Likelihood Multiuser Detection}

Let us define two sets $\mathcal{M}_{1}$ and $\mathcal{M}_{2}$, respectively, for all the possible SM symbols. Assume that BS employs the knowledge about the spreading sequences, and all the channels from the $K$ users to BS. Then, based on (6), the JML-MUD detects the SM symbols of $K$ users as

$$
\begin{aligned}
\{\hat{\boldsymbol{m}}, \hat{\boldsymbol{x}}\}= & \arg \min _{\boldsymbol{m} \in \mathcal{M}_{1}^{K}, \boldsymbol{x} \in \mathcal{M}_{2}^{K}}\left\{\left\|\boldsymbol{y}-\left(\boldsymbol{I}_{U} \otimes \boldsymbol{C}\right) \boldsymbol{H}(\boldsymbol{m}) \boldsymbol{x}\right\|^{2}\right\} \\
= & \arg \max _{\boldsymbol{m} \in \mathcal{M}_{1}^{K}, \boldsymbol{x} \in \mathcal{M}_{2}^{K}}\left\{2 \Re\left\{\boldsymbol{x}^{H} \boldsymbol{H}^{H}(\boldsymbol{m})\left(\boldsymbol{I}_{U} \otimes \boldsymbol{C}^{H}\right) \boldsymbol{y}\right\}\right. \\
& \left.-\left\|\left(\boldsymbol{I}_{U} \otimes \boldsymbol{C}\right) \boldsymbol{H}(\boldsymbol{m}) \boldsymbol{x}\right\|^{2}\right\}
\end{aligned}
$$

Upon invoking the definitions in (5), we can obtain

$$
\begin{aligned}
\{\hat{\boldsymbol{m}}, \hat{\boldsymbol{x}}\}= & \arg \max _{\boldsymbol{m} \in \mathcal{M}_{1}^{K}, \boldsymbol{x} \in \mathcal{M}_{2}^{K}}\left\{\sum _ { u = 0 } ^ { U - 1 } \left(2 \Re\left\{\left[\boldsymbol{C H}_{u}(\boldsymbol{m}) \boldsymbol{x}\right]^{H} \boldsymbol{y}_{u}\right\}\right.\right. \\
& \left.\left.-\left\|\boldsymbol{C} \boldsymbol{H}_{u}(\boldsymbol{m}) \boldsymbol{x}\right\|^{2}\right)\right\}
\end{aligned}
$$

From (8) we are implied that the JML-MUD can be first implemented independently with respect to the $U$ receive antennas, each receive antenna provides $M_{1}^{K} M_{2}^{K}$ decision variables. Then, among the decision variables provided by $U$ receive antennas, the $U$ terms corresponding to the same $m$ and $x$ are added together to give $M_{1}^{K} M_{2}^{K}$ decision variables corresponding to all the possible SM symbols of $K$ users. Finally, the largest one is chosen from the $M_{1}^{K} M_{2}^{K}$ decision variables, from which the SM symbols of $K$ users can be obtained from the corresponding $\boldsymbol{m}$ and $\boldsymbol{x}$.

From (8) we can analyze the complexity of the JML-MUD, which is $\mathcal{O}\left(c U K N L M_{1}^{K} M_{2}^{K}\right)$ or simply $\mathcal{O}\left(c^{\prime} M_{1}^{K} M_{2}^{K}\right)$, where $c$ is a constant determined by the operations of multiplication, addition, etc., of complex numbers, while $c^{\prime}$ is a constant taking into account the computation of matrix (vector) multiplication, addition, etc. This complexity is explicitly extreme for practical implementation, even when a moderate number of users is considered, especially in the case that the constellation size of SM, i.e., $M_{1} M_{2}$, is relatively large. In literature, there are various approaches proposed to reduce the complexity of the JML-MUD at the cost of certain performance loss $[38,51]$. Below we propose three MMSErelying detectors, which have significantly lower complexity than that of the JML-MUD.

\section{B. Minimum Mean-Square Error Aided Spatial Demodulation}

In order to reduce the detection complexity in SM/DSCDMA systems, we propose three detection schemes, namely the MMSE-JSD, MMSE-SSD and the MMSE-IIC. In both the MMSE-JSD and MMSE-SSD, the detection procedure is constituted by two stages. During the first stage, each receive antenna operates a linear MMSE-MUD, attempting to suppress MUI to separate the signals sent by $K$ users. Then, during the second stage, $K$ independent detectors are operated, with each one detecting the SM symbol of one user based on the corresponding signals provided by the $U$ MUDs for that user. Furthermore, in MMSE-JSD, the $M_{1} \mathrm{SSK}$ and $M_{2} \mathrm{APM}$ symbols are jointly demodulated, while in MMSE-SSD, they are separately demodulated. By contrast, MMSE-IIC is an iterative detector, which uses $K$ iterations to detect the $M_{1}$ SSK and $M_{2} \mathrm{APM}$ symbols sent by the $K$ users. Each iteration detects one user rendered the most reliable, whose interference on the later detected users is then cancelled in order to improve these later detected users' performance.

We should emphasize again that the MUD in SM modulated multiuser systems is different from that operated in the conventional multiuser systems, such as, DS-CDMA or SDMA systems $[38,51]$. In the conventional multiuser systems, CSI is usually the a-priori without carrying information, which can be directly exploited for MUI suppression. By contrast, in SM modulated multiuser systems, such as the SM/DS-CDMA in this paper, CSI is dependent on the transmitted information, which is unable to be utilized for MUI suppression before the detection of the $M_{1} \mathrm{SSK}$ symbols. In more detail, from (4) we know that $C$ is a $(N+L-1) \times K L$ matrix, while $\boldsymbol{H}_{u}\left(\boldsymbol{m}_{s}\right)$ is a $(K L \times K)$ matrix, which makes $\boldsymbol{H}_{u}\left(\boldsymbol{m}_{s}\right) \boldsymbol{x}_{s}$ a $K L$-length vector. Since the channel matrix $\boldsymbol{H}_{u}\left(\boldsymbol{m}_{s}\right)$ carries the $M_{1} \mathrm{SSK}$ symbols transmitted by $K$ users, the MUD has to treat $\boldsymbol{H}_{u}\left(\boldsymbol{m}_{s}\right) \boldsymbol{x}_{s}$ as a $K L$-length signal vector to be estimated. In this case, when linear MUD is employed, it in general requires $N+L-1 \geq K L$ or $K \leq(N+L-1) / L$, in order that $C$ is full column rank and, hence, MUI can be efficiently suppressed. Otherwise, the detection performance 
may be poor. By contrast, in the conventional CDMA systems, linear MUDs usually only require $N+L-1 \geq K$ to achieve efficient detection, owing to that the channel matrix is independent of the information transmitted. In our following discourses, however, we derive the MUDs without imposing the above-mentioned constraint of $N+L-1 \geq K L$, in order to demonstrate the impact of the invoked parameters on the achievable performance of the SM/DS-CDMA employing the proposed detectors.

As above-mentioned, both MMSE-JSD and MMSE-SSD are operated in two stages. During the first stage, a MMSE-MUD is operated associated with each of the $U$ receive antennas. The outputs of the $u$ th receive antenna after the MMSE-MUD can be expressed as

$$
\boldsymbol{y}_{u}^{\prime}=\boldsymbol{W}^{H} \boldsymbol{y}_{u}, u=0,1, \ldots, U-1
$$

where $W$ is the weight matrix derived in the sense of MMSE Based on (3) and upon viewing $\boldsymbol{H}_{u}\left(\boldsymbol{m}_{s}\right) \boldsymbol{x}_{s}$ as the signals to be estimated, we can readily show that the weight matrix achieving MMSE-MUD is given by [38]

$$
\boldsymbol{W}=\left(\boldsymbol{C} \boldsymbol{C}^{H}+L \sigma_{s}^{2} \boldsymbol{I}_{N+L-1}\right)^{-1} \boldsymbol{C}
$$

where $\sigma_{s}^{2}=1 / \gamma_{s}$ and the factor $L$ is due to $E\left[\boldsymbol{H}_{u}\left(\boldsymbol{m}_{s}\right) \boldsymbol{x}_{s} \boldsymbol{x}_{s}^{H} \boldsymbol{H}_{u}^{H}\left(\boldsymbol{m}_{s}\right)\right]=\boldsymbol{I}_{K L} / L$. Explicitly, $\boldsymbol{W}$ is constant and is the same for all $U$ receive antennas, provided that the state of active users maintains unchanged. Substituting (3) and (10) into (9) yields

$$
\boldsymbol{y}_{u}^{\prime}=\boldsymbol{C}^{H} \boldsymbol{R}^{-1} \boldsymbol{C} \boldsymbol{H}_{u}\left(\boldsymbol{m}_{s}\right) \boldsymbol{x}_{s}+\boldsymbol{n}_{u}^{\prime},
$$

where, for convenience, we expressed $R=C C^{H}+$ $L \sigma_{s}^{2} \boldsymbol{I}_{N+L-1}$, and $\boldsymbol{n}_{u}^{\prime}=\boldsymbol{C}^{H} \boldsymbol{R}^{-1} \boldsymbol{n}_{u}$.

From (11) we can know that the $L$ terms related to the $k$ th user are given by

$$
\begin{aligned}
\boldsymbol{y}_{u}^{(k)}= & \boldsymbol{C}_{k}^{H} \boldsymbol{R}^{-1} \boldsymbol{C H}_{u}\left(\boldsymbol{m}_{s}\right) \boldsymbol{x}_{s}+\boldsymbol{n}_{u}^{(k)} \\
= & \boldsymbol{C}_{k}^{H} \boldsymbol{R}^{-1} \boldsymbol{C}_{k} \boldsymbol{h}_{m_{k}}^{(k, u)} x_{k}+\sum_{k^{\prime} \neq k}^{K} \boldsymbol{C}_{k}^{H} \boldsymbol{R}^{-1} \boldsymbol{C}_{k^{\prime}} \boldsymbol{h}_{m_{k^{\prime}}}^{\left(k^{\prime}, u\right)} x_{k^{\prime}} \\
& +\boldsymbol{n}_{u}^{(k)}, \quad u=0,1, \ldots, U-1
\end{aligned}
$$

where $\boldsymbol{n}_{u}^{(k)}=\boldsymbol{C}_{k}^{H} \boldsymbol{R}^{-1} \boldsymbol{n}_{u}$, which obeys the Gaussian distribution with zero mean and a covariance matrix of $\boldsymbol{C}_{k}^{H} \boldsymbol{R}^{-2} \boldsymbol{C}_{k} / \gamma_{s}$. Furthermore, from (12) we can deduce that the weight matrix for detecting the $k$ th user is $\boldsymbol{W}_{k}=\boldsymbol{R}^{-1} \boldsymbol{C}_{k}$, which is given by the $L$ columns of $W$ corresponding to user $k$.

Let in (12) $\boldsymbol{A}_{k}=\boldsymbol{C}_{k}^{H} \boldsymbol{R}^{-1} \boldsymbol{C}_{k}$ and $\boldsymbol{i}_{u}^{(k)}=$ $\sum_{k^{\prime} \neq k}^{K} \boldsymbol{C}_{k}^{H} \boldsymbol{R}^{-1} \boldsymbol{C}_{k^{\prime}} \boldsymbol{h}_{m_{k^{\prime}}}^{\left(k^{\prime}, u\right)} x_{k^{\prime}}+\boldsymbol{n}_{u}^{(k)}$. Then, $\boldsymbol{y}_{u}^{(k)}$ can be re-written as

$$
\begin{aligned}
\boldsymbol{y}_{u}^{(k)}=\boldsymbol{A}_{k} \boldsymbol{h}_{m_{k}}^{(k, u)} x_{k}+\boldsymbol{i}_{u}^{(k)}, & u=0,1, \ldots, U-1 \\
k & =1,2, \ldots, K
\end{aligned}
$$

where, according to [53], $\boldsymbol{i}_{u}^{(k)}$ can be approximated as a Gaussian vector, distributed with zero mean and a covariance matrix of

$$
\begin{aligned}
\boldsymbol{R}_{k}= & \frac{1}{L} \sum_{k^{\prime} \neq k}^{K} \boldsymbol{C}_{k}^{H} \boldsymbol{R}^{-1} \boldsymbol{C}_{k^{\prime}} \boldsymbol{C}_{k^{\prime}}^{H} \boldsymbol{R}^{-1} \boldsymbol{C}_{k}+\frac{1}{\gamma_{s}} \boldsymbol{C}_{k}^{H} \boldsymbol{R}^{-2} \boldsymbol{C}_{k}, \\
& k=1,2, \ldots, K
\end{aligned}
$$

Below we consider the MMSE-JSD and MMSE-SSD.
1) MMSE Aided Joint Spatial Demodulation (MMSE-JSD): During the second stage of MMSE-JSD, $K$ joint spatial demodulators (JSDs) are independently operated, each JSD jointly detects the $M_{1} \mathrm{SSK}$ and $M_{2} \mathrm{APM}$ symbols of one user. Specifically, the $M_{1} \mathrm{SSK}$ and $M_{2} \mathrm{APM}$ symbols of the $k$ th user can be jointly detected in ML principle as

$$
\begin{aligned}
&\left\{\hat{m}_{k}, \hat{x}_{k}\right\}= \arg \min _{m_{k} \in \mathcal{M}_{1}, x_{k} \in \mathcal{M}_{2}}\left\{\sum_{u=0}^{U-1}\left(\boldsymbol{y}_{u}^{(k)}-\boldsymbol{A}_{k} \boldsymbol{h}_{m_{k}}^{(k, u)} x_{k}\right)^{H}\right. \\
&\left.\boldsymbol{R}_{k}^{-1}\left(\boldsymbol{y}_{u}^{(k)}-\boldsymbol{A}_{k} \boldsymbol{h}_{m_{k}}^{(k, u)} x_{k}\right)\right\} \\
&=\arg \max _{m_{k} \in \mathcal{M}_{1}, x_{k} \in \mathcal{M}_{2}}\left\{\sum_{u=0}^{U-1} \Re\left\{\left(\boldsymbol{A}_{k} \boldsymbol{h}_{m_{k}}^{(k, u)} x_{k}\right)^{H} \boldsymbol{R}_{k}^{-1} \boldsymbol{y}_{u}^{(k)}\right\}\right. \\
&\left.-\frac{1}{2} \sum_{u=0}^{U-1}\left(\boldsymbol{A}_{k} \boldsymbol{h}_{m_{k}}^{(k, u)}\right)^{H} \boldsymbol{R}_{k}^{-1} \boldsymbol{A}_{k} \boldsymbol{h}_{m_{k}}^{(k, u)}\left|x_{k}\right|^{2}\right\}
\end{aligned}
$$

From (15), we can analyze that the complexity for detecting one user is $\mathcal{O}\left(c U L^{2} M_{1} M_{2}\right)$ and in total for detecting $K$ users is $\mathcal{O}\left(c K U L^{2} M_{1} M_{2}\right)$. Additionally, from (10), we can know that $W$ is a constant matrix, which only needs to be computed once for all the $U$ receive antennas and also for all the $K$ users. It is only required to be updated, when the state of active users changes. Therefore, the complexity contributed by MMSE-MUD is insignificant. Overall, the complexity of MMSE-JSD is linearly dependent on $M_{1}$ and $M_{2}$, and is much lower than that of JML-MUD.

2) MMSE Aided Separate Spatial Demodulation (MMSE$S S D)$ : In order to further reduce the detection complexity, the $M_{1}$ SSK symbol and $M_{2}$ APM symbol of one user may be separately detected. MMSE-SSD follows this detection procedure. In detail, after MMSE-MUD, we have the outputs of (13) for detecting the $M_{1}$ SSK and $M_{2}$ APM symbols of user $k$. In (13), $\boldsymbol{A}_{k}$ is a $(L \times L)$ matrix that is invertible. Therefore, after multiplying (13) with $\boldsymbol{A}_{k}^{-1}$, we obtain

$$
\begin{aligned}
\boldsymbol{z}_{u}^{(k)}=\boldsymbol{h}_{m_{k}}^{(k, u)} x_{k}+\boldsymbol{j}_{u}^{(k)}, & u=0,1, \ldots, U-1 ; \\
k & =1,2, \ldots, K
\end{aligned}
$$

where $\boldsymbol{j}_{u}^{(k)}=\boldsymbol{A}_{k}^{-1} \boldsymbol{i}_{u}^{(k)}$. Based on (16), the $M_{1}$ SSK symbol $m_{k}$ can be estimated as follows. First, we form $M_{1}$ decision variables as

$$
\begin{gathered}
Z_{m}^{(k)}=\left|\sum_{u=0}^{U-1}\left(\boldsymbol{h}_{m}^{(k, u)}\right)^{H} \boldsymbol{z}_{u}^{(k)}\right|, m=0,1, \ldots, M_{1}-1 ; \\
k=1,2, \ldots, K
\end{gathered}
$$

Then, for user $k$, the largest one is chosen from $\left\{Z_{m}^{(k)}\right\}$, whose subscript gives the estimate to $m_{k}$. This can be described as

$$
\begin{gathered}
\hat{m}_{k} \leftrightarrow Z_{\hat{m}_{k}}^{(k)}=\max \left\{Z_{0}^{(k)}, Z_{1}^{(k)}, \cdots, Z_{M_{1}-1}^{(k)}\right\}, \\
k=1,2, \ldots, K
\end{gathered}
$$

After detecting the $M_{1}$ SSK symbol of user $k$, the channel knowledge about user $k$ can be assumed to be known to BS. Hence, BS can use maximal ratio combining (MRC) to detect the $M_{2} \mathrm{APM}$ symbol of user $k$, which can be represented as

$$
\begin{gathered}
\hat{x}_{k}=\operatorname{Demod}_{M_{2} \mathrm{APM}}\left(\sum_{u=0}^{U-1}\left(\boldsymbol{h}_{\hat{m}_{k}}^{(k, u)}\right)^{H} \boldsymbol{z}_{u}^{(k)}\right), \\
k=1,2, \ldots, K
\end{gathered}
$$


where $\operatorname{Demod}_{M_{2} \mathrm{APM}}(x)$ denotes the process of $M_{2}$ APM demodulation [54].

From (17) and (19), we can readily know that the complexity of MMSE-SSD detecting $K$ users is $\mathcal{O}\left(c K U L\left(M_{1}+\right.\right.$ $\left.M_{2}\right)$ ), which is lower than that of MMSE-JSD, provided that $M_{1}, M_{2}>2$. However, as shown in Section $\mathrm{V}$, the performance of MMSE-SSD is worse than that of MMSE-JSD. Furthermore, in the case of $K L>N+L-1$, the performance of both MMSE-JSD and MMSE-SSD is significantly degraded by MUI. Therefore, we below propose the MMSE-IIC, which has a slightly increased complexity than MMSE-JSD, but achieves a significantly enhanced performance.

\section{MMSE-Based Iterative Interference Cancellation}

The MMSE-IIC developed below is based on MMSE-JSD in conjunction with two types of reliability measurement schemes to be proposed. It is well-known that IIC experiences the error propagation problem. For this sake, it is critical that a user detected earlier is more reliable than a user detected later [38]. Hence, we choose MMSE-JSD, first, because it is capable of providing more reliable detection than MMSE$\mathrm{SSD}$, as shown by our performance results in Section V. Second, in both the cases of $(N+L-1) \geq K L$ and $(N+L-1)<K L$, the MMSE-MUD, either MMSE-JSD or MMSE-SSD, in Sections III-B1 and III-B2 have the same form of weight matrix, repeated here as

$$
\begin{gathered}
\boldsymbol{W}_{k}=\boldsymbol{R}^{-1} \boldsymbol{C}_{k}=\left(\boldsymbol{C} \boldsymbol{C}^{H}+L \sigma_{s}^{2} \boldsymbol{I}_{N+L-1}\right)^{-1} \boldsymbol{C}_{k}, \\
k=1,2, \ldots, K
\end{gathered}
$$

which is feasible for implementation. Additionally, we consider JSD instead of SSD, again, in order to mitigate error propagation, owing to the fact that MMSE-JSD outperforms MMSE-SSD in terms of the detection reliability.

As the achievable performance of IIC-type detectors is critically dependent on the accuracy of reliability measurement $[51,55]$, we first propose two reliability measurement schemes. The first one is referred to as joint SM reliability (JSM-Reliability) measurement, while the second one is referred to as the SSK-only reliability (SSKO-Reliability) measurement. In order to derive the JSM-Reliability measurement scheme, we start from the MMSE-JSD considered in Section III-B. According to the analysis in Section III-B, after MMSE-MUD, the processed observations $\boldsymbol{y}^{(k)}=\left[\left(\boldsymbol{y}_{0}^{(k)}\right)^{T}, \cdots,\left(\boldsymbol{y}_{U-1}^{(k)}\right)^{T}\right]^{T}$ for given $m_{k} \in \mathcal{M}_{1}$ and $x_{k} \in \mathcal{M}_{2}$, where $\boldsymbol{y}_{u}^{(k)}$ is given by (12), can be approximated as the Gaussian distributed random vector, with the PDF of

$$
\begin{aligned}
& f\left(\boldsymbol{y}^{(k)} \mid m_{k}, x_{k}\right)=\frac{1}{\left[\pi^{L} \operatorname{det}\left(\boldsymbol{R}_{k}\right)\right]^{U}} \\
& \quad \times \exp \left(-\sum_{u=0}^{U-1}\left[\boldsymbol{y}_{u}^{(k)}-\boldsymbol{A}_{k} \boldsymbol{h}_{m_{k}}^{(k, u)} x_{k}\right]^{H} \boldsymbol{R}_{k}^{-1}\right. \\
& \left.\quad \times\left[\boldsymbol{y}_{u}^{(k)}-\boldsymbol{A}_{k} \boldsymbol{h}_{m_{k}}^{(k, u)} x_{k}\right]\right)
\end{aligned}
$$

The estimates of JSD are given by the $\hat{m}_{k} \in \mathcal{M}_{1}$ and $\hat{x}_{k} \in \mathcal{M}_{2}$, which result in the maximum probability density of $f\left(\boldsymbol{y}^{(k)} \mid \hat{m}_{k}, \hat{x}_{k}\right)$ among the $M_{1} M_{2}$ densities yielded by all $m_{k} \in \mathcal{M}_{1}$ and all $x_{k} \in \mathcal{M}_{2}$. In principle, the estimates of $\hat{m}_{k}$ and $\hat{x}_{k}$ are more reliable, when $f\left(\boldsymbol{y}^{(k)} \mid \hat{m}_{k}, \hat{x}_{k}\right)$ is larger.

Explicitly, the estimates of JSD are equivalently given by the $\hat{m}_{k}$ and $\hat{x}_{k}$, which yield the minimum among the $M_{1} M_{2}$ decision variables of

$$
\begin{aligned}
Z_{i}^{(k)}= & \sum_{u=0}^{U-1}\left(\boldsymbol{y}_{u}^{(k)}-\boldsymbol{A}_{k} \boldsymbol{h}_{m_{k}}^{(k, u)} x_{k}\right)^{H} \boldsymbol{R}_{k}^{-1}\left(\boldsymbol{y}_{u}^{(k)}-\boldsymbol{A}_{k} \boldsymbol{h}_{m_{k}}^{(k, u)} x_{k}\right) \\
& i=0,1, \ldots, M_{1} M_{2}-1
\end{aligned}
$$

where the indices $i \in\left\{0,1, \ldots, M_{1} M_{2}-1\right\}$ correspond to the $M_{1} M_{2}$ combinations between all $m_{k} \in \mathcal{M}_{1}$ and all $x_{k} \in \mathcal{M}_{2}$. Since a smaller value of $Z_{i}^{(k)}$ given by (22) yields a larger density of (21), we can render the estimates of $\hat{m}_{k}$ and $\hat{x}_{k}$ more reliable, if the corresponding $Z_{i}^{(k)}$ is smaller.

When we carefully observe (21), we can realize that the maximum density given by (21) (equivalently, the minimum decision variable of (22)) may not fully reflect the detection reliability. For example, let us consider a SM/DS-CDMA system with $M_{1}=4$ and $M_{2}=2$, and consider two detection cases. In the first case, the densities measured by the detector are 0.5 for one SM symbol of $\left(m_{k}, x_{k}\right)$, while $0.5 / 7$ for all the other $7 \mathrm{SM}$ symbols. By contrast, in the second case, the densities measured by the detector are 0.5 for one SM symbol of $\left(m_{k}, x_{k}\right), 0.49$ for another SM symbol expressed as $\left(m_{k}^{\prime}, x_{k}^{\prime}\right)$, and $0.01 / 6$ for the rest $6 \mathrm{SM}$ symbols. If we measure the reliability based alone on the maximum density, both cases are identified to have the same detection reliability. However, for the above two cases, it is straightforward that the first case has a higher detection reliability than the second one. This is because, in the second case, $\boldsymbol{h}_{m_{k}}^{(k, u)} x_{k}, u=0, \ldots, U-1$ and $\boldsymbol{h}_{m_{k}^{\prime}}^{(k, u)} x_{k}^{\prime}, u=0, \ldots, U-1$ are very close to each other in the Euclidean space, which results in detection confusion.

Therefore, in our first JSM-Reliability measurement scheme, the $k$ th user's detection reliability is measured by the quantity of

$$
\begin{aligned}
\eta_{k}= & \frac{\min \left\{Z_{0}^{(k)}, Z_{1}^{(k)}, \ldots, Z_{M_{1} M_{2}-1}^{(k)}\right\}}{\min _{2}\left\{Z_{0}^{(k)}, Z_{1}^{(k)}, \ldots, Z_{M_{1} M_{2}-1}^{(k)}\right\}} \\
& k=1,2, \ldots, K
\end{aligned}
$$

where $\min \{\cdot\}$ and $\min _{2}\{\cdot\}$ represent, respectively, the minimum and second minimum of the $M_{1} M_{2}$ decision variables given by (22). We render the detection more reliable, when a $\eta_{k}$ is smaller. In other words, the detection of $\hat{m}_{k}$ and $\hat{x}_{k}$ are believed more reliable, if $\min \{\cdot\}$ has a bigger difference from $\min _{2}\{\cdot\}$.

Note that, we prefer (22) to (21), because the computation of (22) is more stable in addition to the reduced computation, while the performance loss is indistinguishable.

During the detection, MMSE-IIC needs to update $\boldsymbol{R}_{k}^{-1}$ for all the users not yet detected, which can be done as follows. Let $\left(\boldsymbol{R}_{k}^{(s-1)}\right)^{-1}$ be the input to the $s$ th iteration of detection, and user $k^{(s)}$ be detected at this iteration. Assume that user $k^{(s)}$ is correctly detected. Then, after the detection, $\left(\boldsymbol{R}_{k}^{(s)}\right)^{-}$ 
for the $k$ th undetected user can be expressed as

$$
\begin{aligned}
\left(\boldsymbol{R}_{k}^{(s)}\right)^{-1}= & \left(\boldsymbol{R}_{k}^{(s-1)}-\frac{1}{L} \boldsymbol{C}_{k}^{H}\left(\boldsymbol{R}^{(s)}\right)^{-1}\right. \\
& \left.\times \boldsymbol{C}_{k^{(s)}} \boldsymbol{C}_{k^{(s)}}^{H}\left(\boldsymbol{R}^{(s)}\right)^{-1} \boldsymbol{C}_{k}\right)^{-1}
\end{aligned}
$$

where $\left(\boldsymbol{R}^{(s)}\right)^{-1}$ is the corresponding update after the $s$ th iteration of detection, which is given later in (30).

From the above analysis, we can know that the JSMReliability measurement first needs to compute $M_{1} M_{2}$ decision variables in the form of (22). Second, it is required to recompute the autocorrelation matrices of the interferenceplus-noise, as shown in (24), for all the users not yet detected. During the detection, a total of $K(K+1) / 2$ autocorrelation matrices in the form of (24) have to be computed. In order to reduce the complexity, below we propose the SSKOReliability measurement scheme, which has lower complexity than the JSM-Reliability measurement but is still highefficiency, especially when $U$ takes a relatively large value.

Following the principles of MMSE-SSD as shown in Section III-B2, when given the processed outputs $\boldsymbol{z}_{u}^{(k)}$ as shown in (16), using the channel knowledge about the $K$ users, $M_{1}$ decision variables can be formed as

$$
Z_{m}^{(k)}=\left|\sum_{u=0}^{U-1}\left(\boldsymbol{h}_{m}^{(k, u)}\right)^{H} \boldsymbol{z}_{u}^{(k)}\right|, m=0,1, \ldots, M_{1}-1
$$

for each of the $K$ users. Then, for each user $k$, the ratio between the second largest and the largest among $\left\{Z_{0}^{(k)}, Z_{1}^{(k)}, \ldots, Z_{M_{1}-1}^{(k)}\right\}$ is evaluated, which is expressed as

$$
\eta_{k}=\frac{\max _{2}\left\{Z_{0}^{(k)}, Z_{1}^{(k)}, \ldots, Z_{M_{1}-1}^{(k)}\right\}}{\max \left\{Z_{0}^{(k)}, Z_{1}^{(k)}, \ldots, Z_{M_{1}-1}^{(k)}\right\}} ; k=1,2, \ldots, K
$$

where $\max _{2}\{\cdot\}$ returns the second maximum of the involved elements. Finally, the user having the minimum ratio is rendered as the most reliable user to be detected.

The rationality behind the SSKO-Reliability measurement scheme lies in the following two facts. First, when the $M_{1}$ SSK symbol is detected with higher reliability, the decision variable of (25) matching to the activated transmit antenna should with high probability be larger than the other $\left(M_{1}-1\right)$ decision variables matching to the inactive transmit antennas. Hence, the ratio of (26) should in general be smaller. Second, when a $M_{2}$ APM symbol is detected with a higher reliability, the SNR for the $M_{2}$ APM detection should be relatively higher. Since the $M_{2} \mathrm{APM}$ symbol is embedded in the variable as

$$
z_{\hat{m}_{1}}^{(k)}=\sum_{u=0}^{U-1}\left(\boldsymbol{h}_{\hat{m}_{1}}^{(k, u)}\right)^{H} \boldsymbol{z}_{u}^{(k)}
$$

its higher detection reliability implies that the decision variable matching to the activated transmit antenna has a relatively larger magnitude. Consequently, the decision variable matching to the activated transmit antenna is most probably larger than the other $\left(M_{1}-1\right)$ decision variables, which match to the inactive transmit antennas. This in turn results in that the ratio of (26) becomes smaller.

In order to describe MMSE-IIC, let $\boldsymbol{R}^{(0)}=$ $\left(\boldsymbol{C} \boldsymbol{C}^{H}+L \sigma_{s}^{2} \boldsymbol{I}_{N+L-1}\right)$, and let $\boldsymbol{R}^{(s)}$ be the autocorrelation matrix generated by the $s$ th iteration with the assumption that all the previous $(s-1)$ iterations provide correct detection. Let us initialize $\boldsymbol{C}^{(0)}=\boldsymbol{C}$ and make $\boldsymbol{C}^{(s)}$ collect all code matrices of the users not yet detected after the $s$ th iteration. Straightforwardly, $\boldsymbol{C}^{(s)}$ is obtained from $\boldsymbol{C}^{(s-1)}$ by removing the $L$ columns corresponding the user detected during the $s$ th iteration, which can be formulated as $C^{(s)}=C^{(s-1)} \boldsymbol{\Phi}^{(s)}$, where $\boldsymbol{\Phi}^{(s)}$ is a mapping matrix obtained from $I_{K L}$ by removing all the columns corresponding to the users having been detected during the zeroth and $s$ th iterations. Then, we have $\boldsymbol{R}^{(s)}=\left[\boldsymbol{C}^{(s)}\left(\boldsymbol{C}^{(s)}\right)^{H}+L \sigma_{s}^{2} \boldsymbol{I}_{N+L-1}\right]$ and $\boldsymbol{W}^{(s)}=\left(\boldsymbol{R}^{(s)}\right)^{-1} \boldsymbol{C}^{(s)}$. Explicitly, we can express

$$
\begin{aligned}
\boldsymbol{R}^{(s)} & =\left[\boldsymbol{C}^{(s-1)}\left(\boldsymbol{C}^{(s-1)}\right)^{H}+L \sigma_{s}^{2} \boldsymbol{I}_{N+L-1}-\boldsymbol{C}_{k^{(s)}} \boldsymbol{C}_{k^{(s)}}^{H}\right] \\
& =\left[\boldsymbol{R}^{(s-1)}-\boldsymbol{C}_{k^{(s)}} \boldsymbol{C}_{k^{(s)}}^{H}\right]
\end{aligned}
$$

Upon applying the matrix inverse lemma, we obtain

$$
\begin{aligned}
& \left(\boldsymbol{R}^{(s)}\right)^{-1}=\left(\boldsymbol{R}^{(s-1)}\right)^{-1}+\left(\boldsymbol{R}^{(s-1)}\right)^{-1} \boldsymbol{C}_{k^{(s)}} \\
& \times\left(\boldsymbol{I}_{L}-\boldsymbol{C}_{k^{(s)}}^{H}\left(\boldsymbol{R}^{(s-1)}\right)^{-1} \boldsymbol{C}_{k^{(s)}}\right)^{-1} \boldsymbol{C}_{k^{(s)}}^{H}\left(\boldsymbol{R}^{(s-1)}\right)^{-1}
\end{aligned}
$$

According to (20), we can express in the above equation $\boldsymbol{W}_{k^{(s)}}^{(s-1)}=\left(\boldsymbol{R}^{(s-1)}\right)^{-1} \boldsymbol{C}_{k^{(s)}}$, which is in fact given by the $L$ columns of $\boldsymbol{W}^{(s-1)}$ corresponding to user $k^{(s)}$. Hence, we have

$$
\begin{aligned}
& \left(\boldsymbol{R}^{(s)}\right)^{-1}=\left(\boldsymbol{R}^{(s-1)}\right)^{-1} \\
& \quad+\boldsymbol{W}_{k^{(s)}}^{(s-1)}\left(\boldsymbol{I}_{L}-\boldsymbol{C}_{k^{(s)}}^{H} \boldsymbol{W}_{k^{(s)}}^{(s-1)}\right)^{-1}\left(\boldsymbol{W}_{k^{(s)}}^{(s-1)}\right)^{H}
\end{aligned}
$$

Furthermore, we have $\boldsymbol{W}^{(s-1)}=\left(\boldsymbol{R}^{(s-1)}\right)^{-1} \boldsymbol{C}^{(s-1)}$. Consequently, multiplying $\boldsymbol{C}^{(s)}$ on both sides of (29) and applying the above results, we have

$$
\begin{aligned}
\boldsymbol{W}^{(s)}= & {\left[\boldsymbol{W}^{(s-1)}+\boldsymbol{W}_{k^{(s)}}^{(s-1)}\left(\boldsymbol{I}_{L}-\boldsymbol{C}_{k^{(s)}}^{H} \boldsymbol{W}_{k^{(s)}}^{(s-1)}\right)^{-1}\right.} \\
& \left.\times \boldsymbol{C}_{k^{(s)}}^{H} \boldsymbol{W}^{(s-1)}\right] \boldsymbol{\Phi}^{(s)}
\end{aligned}
$$

As seen in (31), updating $W_{k^{(s)}}^{(s-1)}$ to $W^{(s)}$ only requires to invert a $(L \times L)$ matrix, instead of inverting a $(N+L-1) \times$ $(N+L-1)$ matrix. Hence, significant reduction of complexity is possible, when $N$ and/or $L$ are large.

With the above preparation, MMSE-IIC algorithm can now be described as follows:

Initialization: $\boldsymbol{y}^{(0)}=\boldsymbol{y}, \boldsymbol{R}^{(0)}=\boldsymbol{R}, \boldsymbol{C}^{(0)}=\boldsymbol{C}, \boldsymbol{W}^{(0)}=$ $\boldsymbol{R}^{-1} \boldsymbol{C}, \boldsymbol{\Phi}^{(0)}=\boldsymbol{I}_{K L}, \boldsymbol{R}_{k}^{(0)}=\boldsymbol{R}_{k}$ for $k=1, \ldots, K$.

Detection and Interference Cancellation:

for $s=1,2, \ldots, K$,

1) Computing the decision variables: $\boldsymbol{z}^{(s)}=$ $\Re\left\{\left(\boldsymbol{W}^{(s-1)}\right)^{H} \boldsymbol{y}^{(s-1)}\right\}$.

2) Finding the most reliable user: For the users $k_{1}^{\prime}, k_{2}^{\prime}, \ldots, k_{K-s+1}^{\prime}$ not detected, compute their reliabilities according to (23), when the JSMReliability measurement is employed, or (26), when the SSKO-Reliability measurement is employed. Then, the most reliable user is given by: $k^{(s)}=\arg \max _{k_{i}^{\prime}}\left\{L_{k_{1}^{\prime}}, L_{k_{2}^{\prime}}, \ldots, L_{k_{K-s+1}^{\prime}}\right\}$. 
3) Detection of the most reliable user: The $M_{1} \mathrm{SSK}$ and $M_{2}$ APM symbols transmitted by the most reliable user is detected based on MMSE-JSD, as described in Section III-B1. Let these detected symbols be expressed as $\hat{m}_{k^{(s)}}$ and $\hat{x}_{k^{(s)}}$.

4) Interference cancellation: $\boldsymbol{y}^{(s)}=\boldsymbol{y}^{(s-1)}-$ $\left(\boldsymbol{I}_{U} \otimes \boldsymbol{C}_{k^{(s)}}\right) \boldsymbol{h}_{\hat{m}^{k}(s)} \hat{x}_{k^{(s)}} ;$

5) Update:

- Updating $\boldsymbol{\Phi}^{(s-1)}$ to $\boldsymbol{\Phi}^{(s)}$ by deleting the $L$ columns related to the $k^{(s)}$ th user;

- Computing $\boldsymbol{W}^{(s)}$ by (31);

- If the JSM-Reliability measurement is employed, computing $\left(\boldsymbol{R}_{k}^{(s)}\right)^{-1}$ for all the users not yet detected by (24).

The proposed MMSE-IIC detectors can be efficiently implemented using the existing algorithms proposed, e.g., in [56]. It can be shown that the complexity of the MMSE-IIC with SSKO-Reliability measurement, referred to as SSKO/MMSEIIC, is on the order of $\mathcal{O}\left(K\left(c_{1} L^{3}+c_{2} M_{1} M_{2}+c_{3} N U L\right)\right)$, where $c_{1}, c_{2}$ and $c_{3}$ are certain constants. Therefore, for a given $N, U$ and $L$, the complexity of SSKO/MMSEIIC increases linearly with $K$. By contrast, the complexity of the MMSE-IIC with the JSM-Reliability measurement, called as JSM/MMSE-IIC, is on the order of $\mathcal{O}\left(K\left(c_{1} L^{3}+c_{2} M_{1} M_{2}+c_{3} N U L\right)+c_{4} K(K+1) L^{3} / 2\right)$, where $c_{1}, c_{2}, c_{3}$ and $c_{4}$ are certain constants. Therefore, for given $N, U$ and $L$, the complexity of JSM/MMSE-IIC increases linearly with $K^{2}$. Furthermore, the detection delay of both MMSE-IIC detectors is also linearly depended on $K$. However, they are symbol-by-symbol detectors, and hence, their maximum detection delay is $K$ symbols.

Finally, the complexity of the MUDs introduced above is summarized in Table I along with the complexity of the MUDs considered in [34] and [33] for the massive MIMO employing SM. Note that, the complexity shown in Table I is the complexity order of the corresponding MUDs, after ignoring the constants determined by the operations of, such as, multiplication, addition, etc. Note furthermore that, in principle, the ordered nearest neighbor search MMSE (ONNS-MMSE) detector proposed in [34] is a search algorithm, which first uses the correlation and MMSE processing aiming to find a set of candidates near the optimum solutions, and then uses the JML principle to find the best possible solution. By contrast, the two detectors proposed in [33], namely the message passing for generalized spatial-modulation (MPGSM) and channel hardening-exploiting message passing for GSM (CHEMP) detectors, belong to the family of iterative parallel interference cancellation.

In both [33] and [34], the massive MIMO scenario is considered, while our paper concerns about the device (machinetype) communications providing high-connectivity. Hence, in $[33,34]$, the value of $U$ (typically $>100$ ) should be much larger than that considered in this paper, which can be one to several. Correspondingly, for a given user, the number of channels to be estimated in massive MIMO systems should be much higher than that to be estimated in our SM/DS-CDMA system. Furthermore, massive MIMO principle requires that the number of users should be significantly lower than $U$. However, overloading multiple-access in device (machine- type) communications is the general requirement. Additionally, [33,34] assume narrow-band fading, without considering the complexity contributed by multipath fading.

\section{ANALYSIS OF ERROR PROBABILITY}

We analyze the average bit error rate (ABER) of SM/DSCDMA systems in two cases. First, we derive the singleuser ABER, when JSD is employed. This ABER serves as the ABER lower-bound as well as the approximate ABER of the SM/DS-CDMA systems supporting multiple users and employing JML-MUD. Then, we derive the approximate ABER of the SM/DS-CDMA systems employing MMSE-JSD.

\section{A. Single-User ABER Bound of SM/DS-CDMA Systems with $J S D$}

The ABER bound of SM with JSD has been analyzed in [14]. Our analysis below follows the procedures in [14]. However, the channels considered in [14] are flat fading channels, while the SM/DS-CDMA systems considered in this paper experience frequency-selective fading. When there is one user, the observation equation of (3) is reduced to

$$
\boldsymbol{y}_{u}=\boldsymbol{C}_{k} \boldsymbol{h}_{m}^{(u)} x+\boldsymbol{n}_{u}, u=0,1, \ldots, U-1
$$

where the index $k$ is ignored for simplicity, when there is no confusion. Correspondingly, the JML-MUD of (33) is reduced to the JSD of

$$
\begin{aligned}
\{\hat{m}, \hat{x}\}= & \arg \max _{m \in \mathcal{M}_{1}, x \in \mathcal{M}_{2}}\left\{\sum _ { u = 0 } ^ { U - 1 } \left[2 \Re\left\{\left(\boldsymbol{C}_{k} \boldsymbol{h}_{m}^{(u)} x\right)^{H} \boldsymbol{y}_{u}\right\}\right.\right. \\
& \left.\left.-\left\|\boldsymbol{C}_{k} \boldsymbol{h}_{m}^{(u)} x\right\|^{2}\right]\right\}
\end{aligned}
$$

Given that $\{\tilde{m}, \tilde{x}\}$ is transmitted, an erroneous symbol $\{\hat{m}, \hat{x}\}$ is detected, provided that there exists at least one test $m \neq \tilde{m}$ and/or $x \neq \tilde{x}$, which makes

$$
\begin{gathered}
\sum_{u=0}^{U-1}\left[2 \Re\left\{\left(\boldsymbol{C}_{k} \boldsymbol{h}_{m}^{(u)} x\right)^{H} \boldsymbol{y}_{u}\right\}-\left\|\boldsymbol{C}_{k} \boldsymbol{h}_{m}^{(u)} x\right\|^{2}\right]> \\
\sum_{u=0}^{U-1}\left[2 \Re\left\{\left(\boldsymbol{C}_{k} \boldsymbol{h}_{\tilde{m}}^{(u)} \tilde{x}\right)^{H} \boldsymbol{y}_{u}\right\}-\left\|\boldsymbol{C}_{k} \boldsymbol{h}_{\tilde{m}}^{(u)} \tilde{x}\right\|^{2}\right]
\end{gathered}
$$

The average probability of this event, referred to as the average pairwise error probability (APEP) can be expressed as [14, 57]

$$
\begin{aligned}
& P_{E P}(\{\tilde{m}, \tilde{x}\} \rightarrow\{m, x\})=E_{\boldsymbol{H}}[Q(\sqrt{2 \gamma})] \\
& \gamma=\sum_{u=0}^{U-1} \gamma_{u}, \gamma_{u}=\frac{\gamma_{s}}{4}\left\|\boldsymbol{C}_{k} \boldsymbol{h}_{m}^{(u)} x-\boldsymbol{C}_{k} \boldsymbol{h}_{\tilde{m}}^{(u)} \tilde{x}\right\|^{2}
\end{aligned}
$$

where $Q(x)=(\sqrt{2 \pi})^{-1} \int_{x}^{\infty} e^{-t^{2} / 2} d t, E_{\boldsymbol{H}}[\cdot]$ means the expectation with respect to all the channels from the considered user to BS. Consequently, the union-bound for the ABER of the single-user SM/DS-CDMA system with JSD can be written as

$$
\begin{aligned}
\bar{P}_{b} \leq & \frac{1}{M_{1} M_{2}\left(b_{1}+b_{2}\right)} \sum_{\tilde{m} \in \mathcal{M}_{1}} \sum_{\tilde{x} \in \mathcal{M}_{2}} \sum_{m \in \mathcal{M}_{1}} \sum_{x \in \mathcal{M}_{2}} \\
& \times D(\{\tilde{m}, \tilde{x}\} ;\{m, x\}) P_{E P}(\{\tilde{m}, \tilde{x}\} \rightarrow\{m, x\})
\end{aligned}
$$


TABLE I

COMPLEXITY OF VARIOUS DETECTORS FOR THE MA SYSTEMS WITH SM.

\begin{tabular}{cccc}
\hline JML-MUD & MMSE-JSD & MMSE-SSD & SSKO/MMSE-IIC \\
\hline $\mathcal{O}\left(M_{1}^{K} M_{2}^{K}\right)$ & $\mathcal{O}\left(K U L^{2} M_{1} M_{2}\right)$ & $\mathcal{O}\left(K U L\left(M_{1}+M_{2}\right)\right)$ & $\mathcal{O}(K N U L)$ \\
\hline \hline JSM/MMSE-IIC & MP-GSM [33] & CHEMP-GSM [33] & O-NNS-MMSE [34] \\
\hline $\mathcal{O}\left(K N U L+K(K+1) L^{3}\right)$ & $\mathcal{O}\left(U K M_{1} M_{2}\right)$ & $\mathcal{O}\left(U K^{2} M_{1}\right)$ & $\mathcal{O}\left(U K^{2}+K^{3}\right)$ \\
\hline
\end{tabular}

where $D(\{\tilde{m}, \tilde{x}\} ;\{m, x\})$ denotes the Hamming distance between the binary representations of $\{\tilde{m}, \tilde{x}\}$ and $\{m, x\}$.

Let $\boldsymbol{g}_{u}=\boldsymbol{C}_{k} \boldsymbol{h}_{m}^{(u)} x-\boldsymbol{C}_{k} \boldsymbol{h}_{\tilde{m}}^{(u)} \tilde{x}$, which is a $(N+L-1)$ length complex vector, and for given $C_{k}, x$ and $\tilde{x}$, obeys the circularly-symmetric Gaussian distribution. Furthermore, $\gamma_{u}$ in (35b) can be written as

$$
\gamma_{u}=\frac{\gamma_{s}}{4} \sum_{n=0}^{N+L-2}\left|g_{u}(n)\right|^{2}
$$

In $\boldsymbol{g}_{u}$, both $\boldsymbol{h}_{m}^{(u)}$ and $\boldsymbol{h}_{\tilde{m}}^{(u)}$ are circularly-symmetric Gaussian distributed. Hence, $\boldsymbol{g}_{u}(n)$ in (37) are complex Gaussian random variables, which make $\gamma_{u}$ obey the Gamma distribution. However, the elements of $\boldsymbol{g}_{u}$ are correlated, but $\gamma_{u}$ in terms of different $u$ are independent and obey identical distribution. Based on the above analysis and the analysis in $[38,58,59]$, it can be shown that the APEP of (35a) has the form of

$$
\begin{aligned}
& P_{E P}(\{\tilde{m}, \tilde{x}\} \rightarrow\{m, x\}) \\
& \quad=\frac{1}{\pi} \int_{0}^{\pi / 2}\left[\operatorname{det}\left(\boldsymbol{I}_{N+L-1}+\frac{\gamma_{s}}{2 \sin \theta} \boldsymbol{M}\right)\right]^{-U} d \theta
\end{aligned}
$$

where $\operatorname{det}(\cdot)$ denotes the determinant, and $M$ is the covariance matrix of $\Re\left\{\boldsymbol{g}_{u}\right\}$ (or $\Im\left\{\boldsymbol{g}_{u}\right\}$ ), expressed as

$$
\boldsymbol{M}=E_{\boldsymbol{H}}\left[\Re\left\{\boldsymbol{g}_{u}\right\}\left(\Re\left\{\boldsymbol{g}_{u}\right\}\right)^{T}\right]
$$

Equation (38) shows that the APEP can be computed provided that $M$ is given. Below we derive $M$ in the context of three cases. In the first case, we assume that the $M_{1}$ SSK symbol is detected in error, while the $M_{2}$ APM symbol is correctly detected. In this case, we have

$$
\begin{aligned}
\boldsymbol{g}_{u} & =\boldsymbol{C}_{k}\left(\boldsymbol{h}_{m}^{(u)}-\boldsymbol{h}_{\tilde{m}}^{(u)}\right) \tilde{x} \\
\boldsymbol{M}_{1}(\tilde{x}) & =\frac{1}{2} \Re\left\{E_{\boldsymbol{H}}\left[\boldsymbol{g}_{u} \boldsymbol{g}_{u}^{H}\right]\right\}=\frac{|\tilde{x}|^{2}}{L} \Re\left\{\boldsymbol{C}_{k} \boldsymbol{C}_{k}^{H}\right\}
\end{aligned}
$$

In the context of the second case, we assume that the $M_{1}$ SSK symbol is correctly detected, while the $M_{2}$ APM symbol is detected in error. Then, we have

$$
\begin{aligned}
\boldsymbol{g}_{u} & =\boldsymbol{C}_{k} \boldsymbol{h}_{\tilde{m}}^{(u)}(x-\tilde{x}) \\
\boldsymbol{M}_{2}(x, \tilde{x}) & =\frac{|x-\tilde{x}|^{2}}{2 L} \Re\left\{\boldsymbol{C}_{k} \boldsymbol{C}_{k}^{H}\right\}
\end{aligned}
$$

Finally, in the third case when both the $M_{1}$ SSK and $M_{2} \mathrm{APM}$ symbols are simultaneously detected in error, we have

$$
\begin{aligned}
\boldsymbol{g}_{u} & =\boldsymbol{C}_{k} \boldsymbol{h}_{m}^{(u)} x-\boldsymbol{C}_{k} \boldsymbol{h}_{\tilde{m}}^{(u)} \tilde{x} \\
\boldsymbol{M}_{3}(x, \tilde{x}) & =\frac{|x|^{2}+|\tilde{x}|^{2}}{2 L} \Re\left\{\boldsymbol{C}_{k} \boldsymbol{C}_{k}^{H}\right\}
\end{aligned}
$$

When taking the above three cases into account, and considering the facts that $M_{1}(\tilde{x}), M_{2}(x, \tilde{x})$ and $M_{3}(x, \tilde{x})$ are all independent of $m$ and $\tilde{m}$, the ABER bound of (36) can be expressed as

$$
\begin{aligned}
\bar{P}_{b} \leq & \frac{M_{1} b_{1}}{2 M_{2}\left(b_{1}+b_{2}\right)} \sum_{\tilde{x} \in \mathcal{M}_{2}} \\
& \times \frac{1}{\pi} \int_{0}^{\pi / 2}\left[\operatorname{det}\left(\boldsymbol{I}_{N+L-1}+\frac{\gamma_{s}}{2 \sin ^{2} \theta} \boldsymbol{M}_{1}(\tilde{x})\right)\right]^{-U} d \theta \\
+ & \frac{1}{M_{2}\left(b_{1}+b_{2}\right)} \sum_{\tilde{x} \in \mathcal{M}_{2}} \sum_{x \neq \tilde{x}} D(\tilde{x} ; x) \\
& \times \frac{1}{\pi} \int_{0}^{\pi / 2}\left[\operatorname{det}\left(\boldsymbol{I}_{N+L-1}+\frac{\gamma_{s}}{2 \sin ^{2} \theta} \boldsymbol{M}_{2}(x, \tilde{x})\right)\right]^{-U} d \theta \\
+ & \frac{1}{2 M_{2}\left(b_{1}+b_{2}\right)} \sum_{\tilde{x} \in \mathcal{M}_{2}} \sum_{x \neq \tilde{x}}\left[M_{1} b_{1}+2\left(M_{1}-1\right) D(\tilde{x} ; x)\right] \\
& \times \frac{1}{\pi} \int_{0}^{\pi / 2}\left[\operatorname{det}\left(\boldsymbol{I}_{N+L-1}+\frac{\gamma_{s}}{2 \sin ^{2} \theta} \boldsymbol{M}_{3}(x, \tilde{x})\right)\right]^{-U} d \theta
\end{aligned}
$$

In the derivation of the above expressions, we applied $b_{1} M_{1} /\left(2 M_{1}-2\right)$ as the average number of erroneous bits per $M_{1}$ SSK symbol [54], whenever an error detection occurs with the $M_{1}$ SSK detection.

Furthermore, in (41), (43) and (45), $\boldsymbol{C}_{k}$ is full column rank and, hence, $\Re\left\{\boldsymbol{C}_{k} \boldsymbol{C}_{k}^{H}\right\}$ has $L$ nonzero eigenvalues. Let these nonzero eigenvalues be expressed as $\lambda_{0}, \ldots, \lambda_{L-1}$. Then, it can be shown that (46) can be expressed as

$$
\begin{aligned}
\bar{P}_{b} \leq & \frac{M_{1} b_{1}}{2 M_{2}\left(b_{1}+b_{2}\right)} \sum_{\tilde{x} \in \mathcal{M}_{2}} \\
& \times \frac{1}{\pi} \int_{0}^{\pi / 2} \prod_{l=0}^{L-1}\left(\frac{2 \sin ^{2} \theta}{2 \sin ^{2} \theta+\lambda_{l} \gamma_{s}|\tilde{x}|^{2} / L}\right)^{U} d \theta \\
+ & \frac{1}{M_{2}\left(b_{1}+b_{2}\right)} \sum_{\tilde{x} \in \mathcal{M}_{2}} \sum_{x \neq \tilde{x}} D(\tilde{x} ; x) \\
& \times \frac{1}{\pi} \int_{0}^{\pi / 2} \prod_{l=0}^{L-1}\left(\frac{4 \sin ^{2} \theta}{4 \sin ^{2} \theta+\lambda_{l} \gamma_{s}|x-\tilde{x}|^{2} / L}\right)^{U} d \theta \\
+ & \frac{1}{2 M_{2}\left(b_{1}+b_{2}\right)} \sum_{\tilde{x} \in \mathcal{M}_{2}} \sum_{x \neq \tilde{x}}\left[M_{1} b_{1}+2\left(M_{1}-1\right) D(\tilde{x} ; x)\right] \\
& \times \frac{1}{\pi} \int_{0}^{\pi / 2} \prod_{l=0}^{L-1}\left(\frac{4 \sin ^{2} \theta}{4 \sin ^{2} \theta+\lambda_{l} \gamma_{s}\left(|x|^{2}+|\tilde{x}|^{2}\right) / L}\right)^{U} d \theta
\end{aligned}
$$

which explicitly show that the JSD is capable of attaining $L U$ orders of diversity, with $U$ orders corresponding to the $U$ receive antennas, and $L$ orders reflecting the $L$-tap frequencyselective fading.

As a demonstration, when $L=1, \Re\left\{\boldsymbol{C}_{k} \boldsymbol{C}_{k}^{H}\right\}$ has only one 
nonzero eigenvalue of one. In this case, (47) is reduced to

$$
\begin{aligned}
\bar{P}_{b} \leq & \frac{M_{1} b_{1}}{2 M_{2}\left(b_{1}+b_{2}\right)} \sum_{\tilde{x} \in \mathcal{M}_{2}} \frac{1}{\pi} \int_{0}^{\pi / 2}\left(\frac{2 \sin ^{2} \theta}{2 \sin ^{2} \theta+\gamma_{s}|\tilde{x}|^{2}}\right)^{U} d \theta \\
+ & \frac{1}{M_{2}\left(b_{1}+b_{2}\right)} \sum_{\tilde{x} \in \mathcal{M}_{2}} \sum_{x \neq \tilde{x}} D(\tilde{x} ; x) \\
& \times \frac{1}{\pi} \int_{0}^{\pi / 2}\left(\frac{4 \sin ^{2} \theta}{4 \sin ^{2} \theta+\gamma_{s}|x-\tilde{x}|^{2}}\right)^{U} d \theta \\
+ & \frac{1}{2 M_{2}\left(b_{1}+b_{2}\right)} \sum_{\tilde{x} \in \mathcal{M}_{2}} \sum_{x \neq \tilde{x}}\left[M_{1} b_{1}+2\left(M_{1}-1\right) D(\tilde{x} ; x)\right] \\
& \times \frac{1}{\pi} \int_{0}^{\pi / 2}\left(\frac{4 \sin ^{2} \theta}{4 \sin ^{2} \theta+\gamma_{s}\left(|x|^{2}+|\tilde{x}|^{2}\right)}\right)^{U} d \theta
\end{aligned}
$$

which is the ABER bound provided in [14] for the SM systems experiencing flat Rayleigh fading.

\section{B. ABER of SM/DS-CDMA Systems with MMSE-JSD}

To derive the ABER of the SM/DS-CDMA systems with MMSE-JSD, we express (12) as

$$
\boldsymbol{y}_{u}^{(k)}=\boldsymbol{A} \boldsymbol{h}_{m}^{(u)} x+\boldsymbol{i}_{u}, u=0,1, \ldots, U-1
$$

where, again, the index $k$ is neglected, when there is no confusion. As defined below (12), in (49), $A=$ $\boldsymbol{C}_{k}^{H} \boldsymbol{R}^{-1} \boldsymbol{C}_{k}$ and $\boldsymbol{i}_{u}=\sum_{k^{\prime} \neq k}^{K} \boldsymbol{C}_{k}^{H} \boldsymbol{R}^{-1} \boldsymbol{C}_{k^{\prime}} \boldsymbol{h}_{m_{k^{\prime}}}^{\left(k^{\prime}, u\right)} x_{k^{\prime}}+\boldsymbol{n}_{u}^{(k)}$ where $\boldsymbol{i}_{u}$ can be approximated as a Gaussian vector, distributed with zero mean and a covariance matrix of $\boldsymbol{R}_{k}=$ $L^{-1} \sum_{k^{\prime} \neq k}^{K} \boldsymbol{C}_{k}^{H} \boldsymbol{R}^{-1} \boldsymbol{C}_{k^{\prime}} \boldsymbol{C}_{k^{\prime}}^{H} \boldsymbol{R}^{-1} \boldsymbol{C}_{k}+\boldsymbol{C}_{k}^{H} \boldsymbol{R}^{-2} \boldsymbol{C}_{k} / \gamma_{s}$. When $\left\{\boldsymbol{C}_{k}\right\}$ are known, both $\boldsymbol{A}$ and $\boldsymbol{R}_{k}$ in (49) are fixed matrices.

Then, when JSD is employed, $\left\{\hat{m}_{k}, \hat{x}_{k}\right\}$ is detected in the ML principle as in (15). Therefore, given that $\{\tilde{m}, \tilde{x}\}$ is transmitted by user $k$, an erroneous symbol $\{\hat{m}, \hat{x}\}$ is detected, when there exists at least one test $m \neq \tilde{m}$ and/or $x \neq \tilde{x}$, which makes

$$
\begin{aligned}
\sum_{u=0}^{U-1} & {\left[2 \Re\left\{\left(\boldsymbol{A} \boldsymbol{h}_{m}^{(u)} x\right)^{H} \boldsymbol{R}_{k}^{-1} \boldsymbol{y}_{u}^{(k)}\right\}\right.} \\
& \left.-\left(\boldsymbol{A} \boldsymbol{h}_{m}^{(u)} x\right)^{H} \boldsymbol{R}_{k}^{-1} \boldsymbol{A} \boldsymbol{h}_{m}^{(u)} x\right]> \\
& \sum_{u=0}^{U-1}\left[2 \Re\left\{\left(\boldsymbol{A} \boldsymbol{h}_{\tilde{m}}^{(u)} \tilde{x}\right)^{H} \boldsymbol{R}_{k}^{-1} \boldsymbol{y}_{u}^{(k)}\right\}\right. \\
& \left.-\left(\boldsymbol{A} \boldsymbol{h}_{\tilde{m}}^{(u)} \tilde{x}\right)^{H} \boldsymbol{R}_{k}^{-1} \boldsymbol{A} \boldsymbol{h}_{\tilde{m}}^{(u)} \tilde{x}\right]
\end{aligned}
$$

Since $\boldsymbol{R}_{k}$ is a covariance matrix, it is a Hermitian matrix and its inverse can be decomposed as $\boldsymbol{R}_{k}^{-1}=$ $\left(\boldsymbol{R}_{k}^{-1 / 2}\right)^{H} \boldsymbol{R}_{k}^{-1 / 2}$ [51]. With the aid of this property, we can show that the APEP between $\{\tilde{m}, \tilde{x}\}$ and $\{m, x\}$ can be expressed as (35a) associated with

$$
\gamma=\sum_{u=0}^{U-1} \gamma_{u}, \gamma_{u}=\frac{1}{4}\left\|\boldsymbol{R}_{k}^{-1 / 2} \boldsymbol{A}\left(\boldsymbol{h}_{m}^{(u)} x-\boldsymbol{h}_{\tilde{m}}^{(u)} \tilde{x}\right)\right\|^{2}
$$

Therefore, following the same procedure for analyzing the single-user ABER in Section IV-A and treating $\boldsymbol{R}_{k}^{-1 / 2} \boldsymbol{A}$ as
$\boldsymbol{C}_{k}$, we can obtain

$$
\begin{aligned}
\boldsymbol{M}_{1}(\tilde{x}) & =\frac{|\tilde{x}|^{2}}{L} \Re\left\{\boldsymbol{R}_{k}^{-1 / 2} \boldsymbol{A}\left(\boldsymbol{R}_{k}^{-1 / 2} \boldsymbol{A}\right)^{H}\right\} \\
\boldsymbol{M}_{2}(x, \tilde{x}) & =\frac{|x-\tilde{x}|^{2}}{2 L} \Re\left\{\boldsymbol{R}_{k}^{-1 / 2} \boldsymbol{A}\left(\boldsymbol{R}_{k}^{-1 / 2} \boldsymbol{A}\right)^{H}\right\} \\
\boldsymbol{M}_{3}(x, \tilde{x}) & =\frac{|x|^{2}+|\tilde{x}|^{2}}{2 L} \Re\left\{\boldsymbol{R}_{k}^{-1 / 2} \boldsymbol{A}\left(\boldsymbol{R}_{k}^{-1 / 2} \boldsymbol{A}\right)^{H}\right\}
\end{aligned}
$$

Let the $L$ nonzero eigenvalues of $\Re\left\{\boldsymbol{R}_{k}^{-1 / 2} \boldsymbol{A}\left(\boldsymbol{R}_{k}^{-1 / 2} \boldsymbol{A}\right)^{H} / \gamma_{s}\right\}$ be expressed as $\lambda_{0}, \ldots, \lambda_{L-1}$. Then, the approximate ABER of the SM/DS-CDMA systems with MMSE-JSD can be expressed as (47). Let us below provide some results to illustrate the achievable error performance of the SM/DS-CDMA systems employing different detection strategies.

\section{Performance Results}

In this section, we first show the BER performance of SM/DS-CDMA systems in Section V-A. Then in Section V-B, we compare the BER performance of SM/DS-CDMA with that of two existing schemes from references. Note that in the figures, the parameters used for defining systems and channel models are detailed. Furthermore, the APM used in our simulations is the QAM with Gray mapping [54], and, unless explicitly notified, the 'Average SNR per bit' means the average SNR per bit per receive antenna.

\section{A. BER Performance of SM/DS-CDMA}

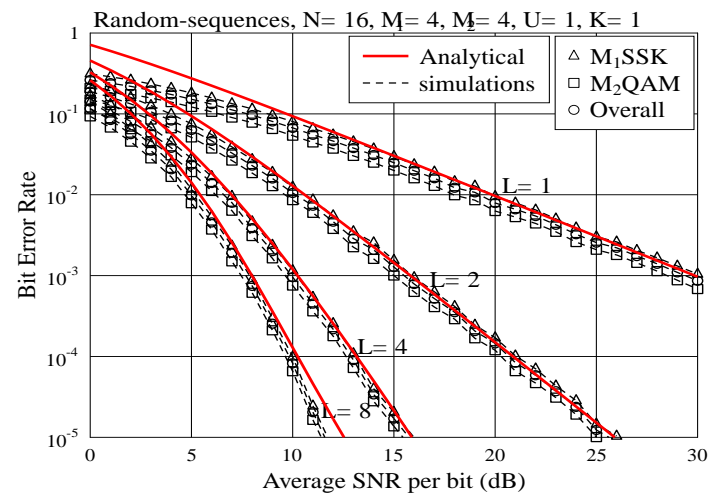

Fig. 3. Single-user BER performance of SM/DS-CDMA systems employing random sequences and JSD.

In the first set of BER results shown in Figs. 3, 4 and 5, we assume that the SM/DS-CDMA system supports only one user and employs the optimum JSD, in order to show the potential performance achievable by SM/DS-CDMA systems, as well as to investigate the impact of the system parameters on the BER performance. Specifically, in order to highlight the frequency diversity gain achievable by SM/DS-CDMA systems, in Fig. 3, we set $U=1$. Explicitly, the BER performance improves, when the number of T-domain resolvable paths increases, implying that SM/DS-CDMA systems are capable of achieving higher frequency diversity gain. From (47) we can know that the order of frequency diversity achievable is equal to the number of T-domain resolvable paths, which is explicitly reflected by the BER performance shown in Fig. 3. 


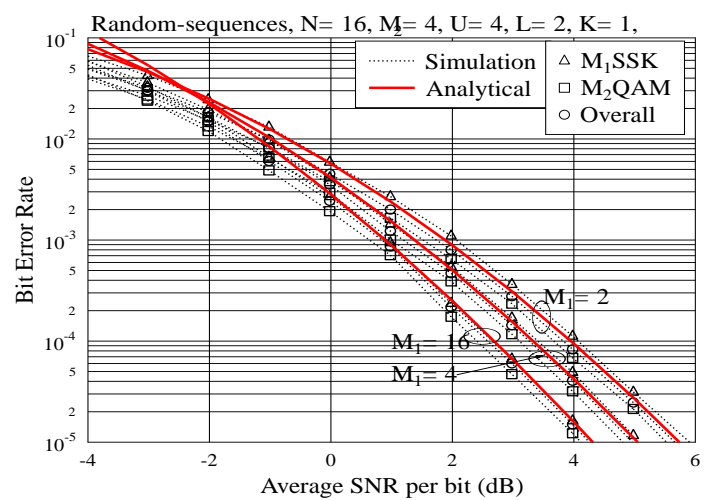

Fig. 4. Single-user BER performance of SM/DS-CDMA systems employing JSD.

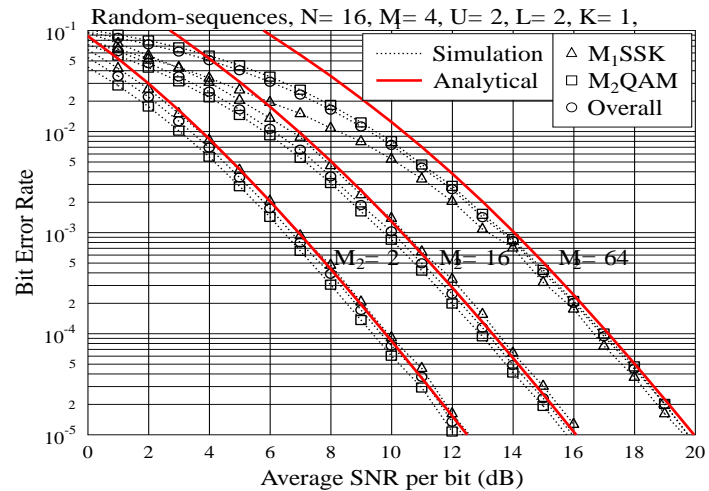

Fig. 5. Single-user BER performance of SM/DS-CDMA systems employing JSD.

In both Figs. 4 and 5, we illustrate the impacts of the modulation levels of $M_{1} \mathrm{SSK}$ and $M_{2} \mathrm{QAM}$ on the BER performance of SM/DS-CDMA systems. As shown in Fig. 4, given the $M_{2} \mathrm{QAM}$ and when the SNR is relatively high (such as, larger than $-1 \mathrm{~dB}$ ), the BER performance of SM/DSCDMA systems slightly improves, as the modulation level $M_{1}$ of $M_{1} \mathrm{SSK}$ increases. The reason behind this observation is that the index-based modulation scheme $M_{1} \mathrm{SSK}$ is an energy-efficient modulation scheme [54]. By contrast, Fig. 5 shows that the BER performance of SM/DS-CDMA systems significantly degrades with the increase of the modulation level $M_{2}$ of $M_{2} \mathrm{QAM}$, as the result that QAM belongs to the family of bandwidth-efficient modulation schemes [54], which are not energy-efficient. Therefore, when given a total number of bits transmitted per SM/DS-CDMA symbol, we may assign more bits to $M_{1}$ SSK modulation and fewer bits to $M_{2} \mathrm{QAM}$, in order to achieve better BER performance. However, this arrangement needs to cost more resource from transmit antennas, which otherwise may be used for different purpose, such as for achieving transmit diversity. Finally, in these three figures, the BER performance of $M_{1} \mathrm{SSK}$, that of $M_{2} \mathrm{QAM}$, as well as the overall BER performance of SM/DS-CDMA systems are respectively illustrated. Explicitly, they are very close to each other, implying that the reliability of detecting $M_{1}$ SSK symbols and that of detecting $M_{2} \mathrm{QAM}$ symbols are generally dependent on each other.

The second set of results include Figs. 6 and 7, where we show and compare the BER performance of the SM/DSCDMA systems employing the MMSE-JSD, JSM/MMSE-

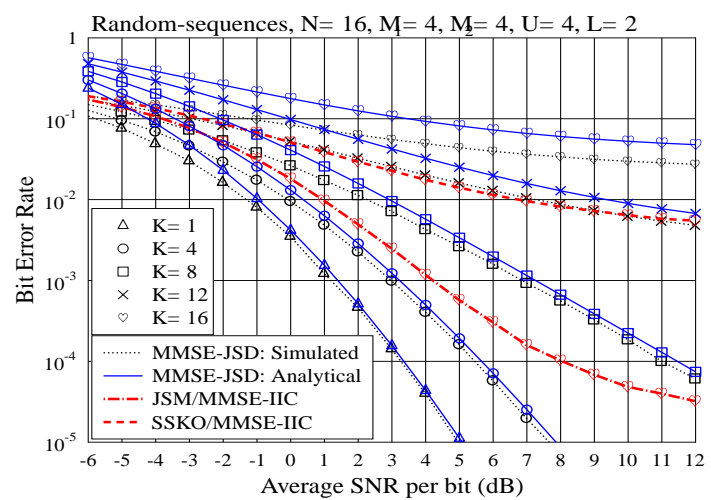

Fig. 6. BER performance of SM/DS-CDMA systems employing respectively the MMSE-JSD, JSM/MMSE-IIC and SSKO/MMSE-IIC, respectively.

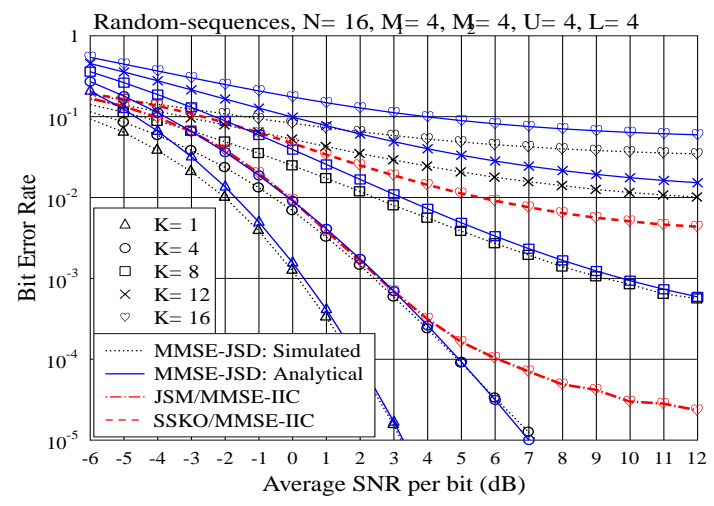

Fig. 7. BER performance of SM/DS-CDMA systems employing respectively the MMSE-JSD and MMSE-IIC.

IIC and the SSKO/MMSE-IIC, respectively. As shown in the figures, for the MMSE-JSD, we consider $K=1,4,8,12,16$, while for the MMSE-IIC, we only consider the full-load case of $K=16$. Furthermore, in both Fig. 6 and Fig. 7, the ABER of the SM/DS-CDMA systems employing the MMSEJSD evaluated from the formulas derived in Section IV-B are provided. Explicitly, the ABER bounds can provide a tight approximation for the corresponding BER of the SM/DSCDMA systems, especially when the systems are operated in a relatively high SNR region, resulting in that the BER is below $10^{-2}$. From Fig. 6 and Fig. 7, we observe that when the condition of $K \leqslant(N+L-1) / L$ is not satisfied, error floor appears. Hence, when comparing Fig. 7 with Fig. 6 for given $K$ and $N$, increasing $L$ although provides higher frequency diversity gain, yielding BER improvement in low SNR region, it also imposes severer MUI, resulting in the BER degradation in relatively high SNR region. Finally, as shown in Figs. 6 and 7, both MMSE-IIC detectors significantly outperform the MMSE-JSD, while the JSM/MMSE-IIC is capable of achieving much better BER performance than the SSKO/MMSE-IIC.

In Fig. 8, the BER performance of the SM/DS-CDMA systems employing MMSE-SSD is studied. Since the MMSESSD successively detects the SSK symbol and QAM symbol, the detection performance of QAM is greatly relied on that of SSK. In order to show this effect, we simply let $K=1$. From Fig. 8 we observe that error floors present, when $L \times U=8$ and 16 . The reason behind is that, when $L \times U$ is relatively small, the SSK's detection given by (17) is not optimal and 


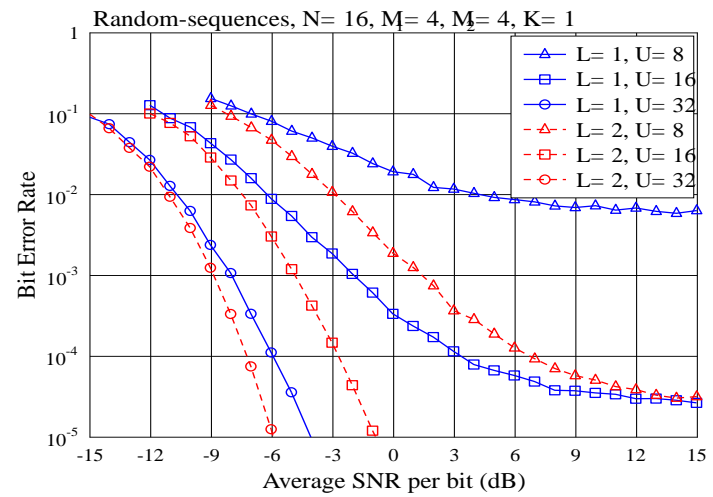

Fig. 8. BER performance of SM/DS-CDMA systems employing MMSESSD.

hence less reliable due to the correlation existing among the channels from different transmit antennas. Furthermore, this less reliable detection of SSK results in channel's mismatch for the following QAM detection using (19). By contrast, when the $L \times U$ is relatively large, according to the principles of massive MIMO [20,26,33,34], the channel vectors in terms of different transmit antennas tend to become orthogonal. Therefore, the SSK detection of (17) converges to an optimum detector [54], which enhances the following QAM detection and makes error floors disappear in the BER range of interest. Additionally, we observe that the curve corresponding to $L=2$ and $U=16$ with that corresponding to $L=1$ and $U=32$ are nearly in parallel, which means that the same diversity gain is achieved by both cases. In other words, the frequency diversity and spatial diversity are equivalent to each other in SM/DS-CDMA systems, when there is single user supported.

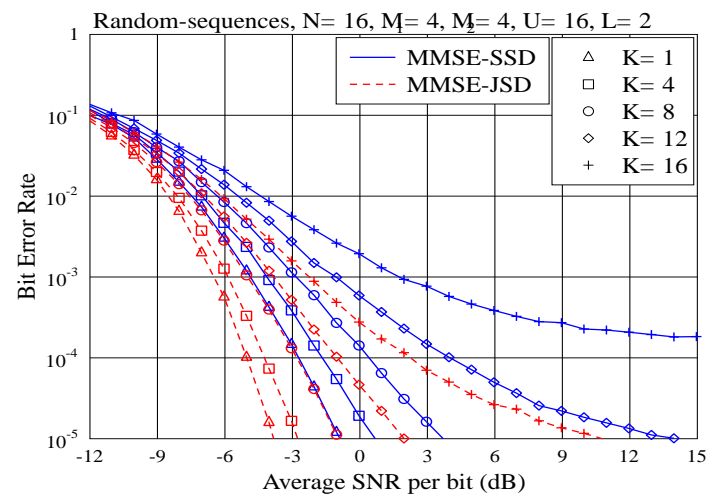

Fig. 9. Comparison of BER performance of the SM/DS-CDMA systems employing MMSE-JSD and MMSE-SSD, respectively.

The third set of results, including Figs. 9 and 10, compare the BER performance of the SM/DS-CDMA systems employing various MMSE-based MUDs. Specifically, the comparison between MMSE-JSD and MMSE-SSD is depicted in Fig. 9, showing that the MMSE-JSD significantly outperforms the MMSE-SSD in terms of BER performance, but at the cost of higher implementation complexity, as shown by the analysis in Sections III-B1 and III-B2. By contrast, in Fig. 10, we compare the BER performance of the SM/DS-CDMA systems employing the JSM/MMSE-IIC and SSKO/MMSE-IIC, respectively. Explicitly, the JSM/MMSE-IIC significantly outperforms the

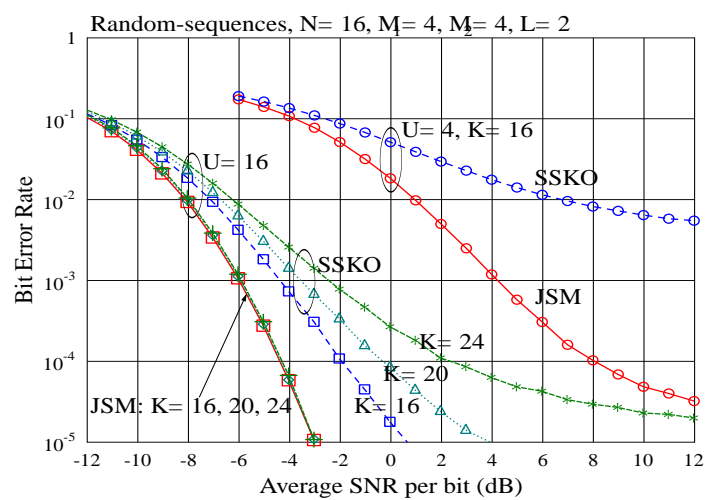

Fig. 10. Comparison of BER performance of the SM/DS-CDMA systems employing JSM/MMSE-IIC and SSKO/MMSE-IIC, respectively.

SSKO/MMSE-IIC, at a cost of higher implementation complexity. As shown in Fig. 10, when $U=16$, both the MMSEIIC schemes are capable of effectively suppressing MUI, even when the SM/DS-CDMA systems are overload in terms of $N$, i.e., $K / N>1$. Furthermore, by comparing the curve of the MMSE-JSD with $K=1$ shown in Fig. 9 with that of the JSM/MMSE-IIC, we can observe that the JSM/MMSE-IIC is capable of achieving near single user performance, even when $K / N=24 / 16=1.5$. Additionally, when $U=4$ and when the system is full load, the JSM/MMSE-IIC is still effective, if the desired BER is in the region of $\left[10^{-4}, 10^{-2}\right]$. However, due to its in incapability of MUI suppression, the SSKO/MMSE-IIC is not effective within this desired BER region.

\section{B. Comparison with Legacy Schemes}

In this subsection, we compare our schemes with two existing schemes from references. Specifically, the SM-SDMA scheme addressed in [31] and the MP-GSM detector proposed in [33] are considered.

Fig. 11 compares the BER performance of SM/DS-CDMA with that of the SM-SDMA in [31], when both of them employ JML-MUD. In order to make a fair comparison, we assume that the product of the spreading factor and the number of receive antennas in SM/DS-CDMA equals the number of receive antennas in SM-SDMA, i.e., the SM-SDMA system employs $N \times U$ receive antennas ${ }^{3}$. The 'Average SNR per bit' in Fig. 11 represents the average SNR per bit normalized by the number of receive antennas. We assume that signals in SMSDMA system experience flat fading, corresponding to $L=1$, while signals in SM/DS-CDMA system experience multipath fading with $L=N$ paths, as the result that the bandwidth of SM/DS-CDMA is $N$ times of that of SM-SDMA, when a given data rate is transmitted.

From Fig. 11, it can be observed that, when $N \times U$ is relatively large, such as 64 , which is practically true when high connectivity is motivated, the two schemes achieve nearly the same BER performance. By contrast, when $N \times U$ is small, the BER performance of SM-SDMA system is slightly better than that of SM/DS-CDMA system. However, the SM-SDMA scheme does not perform well in low SNR region, and its BER

\footnotetext{
${ }^{3}$ Therefore, the $N U$ resource units of SM/DS-CDMA are from a joint space-frequency plane, while of SM-SDMA are solely from the space dimension.
} 


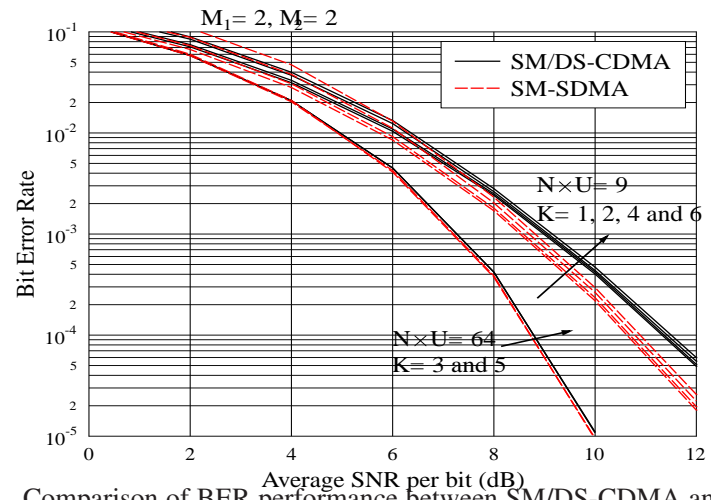

Fig. 11. Comparison of BER performance between SM/DS-CDMA and SMSDMA systems, when $N \times U=3 \times 3=9$ and $N \times U=8 \times 8=64$ are considered, respectively, where the arrow indicates the increase of $K$.

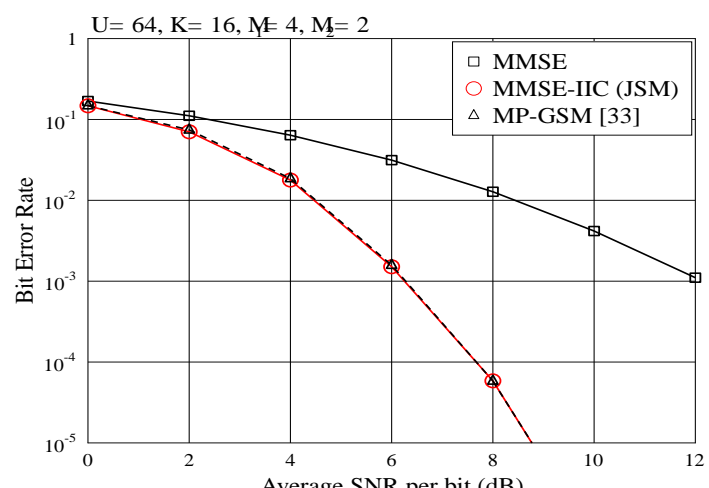

Fig. 12. Comparison of BER performance of the SM-SDMA systems employing the JSM/MMSE-IIC and MPA-MUD [33], respectively.

performance may be slightly worse than that of the SM/DSCDMA scheme (as seen in Fig. 11, when the BER is above 0.01 in the case of $K=6$ ). Furthermore, the SM-SDMA scheme is more sensitive to the number of users supported than the SM/DS-CDMA scheme. As shown in Fig. 11 for the case of $N \times U=9$, the BER performance of SM-SDMA degrades noticeably, while that of SM/DS-CDMA maintains nearly the same, as the number of users increases from $K=1$ to $K=6$. From our previous discourses, we can recall that in SM/DS-CDMA systems, using T-domain spreading codes facilitates to achieve the low-complexity MUDs, where the high-complexity matrix inverse is independent of channels' states, and is required to be updated only when the state of active users changes. By contrast, in the SM-SDMA types of systems, this type of high-complexity computation has to be updated, whenever the channels or the state of active users change. Considering that a SM-SDMA system requires a big number of antennas to support high-connectivity, making the overall channel highly time-variant, the burden of the MUD-related computation should be extreme, in addition to the heavy burden imposed on channel estimation. Therefore, in contrast to SM-SDMA, SM/DS-CDMA is more feasible for supporting high-connectivity MA. Finally, the comparison between SM-SDMA and SM/DS-CDMA is summarized in Table II, explicitly showing the trade-off between the two schemes.

Fig. 12 compares the BER performance of the SM-SDMA systems employing, respectively, the JSM/MMSE-IIC, the MP-GSM detector proposed in [33], and the MMSE-MUD.
The complexity of the JSM/MMSE-IIC and MP-GSM detectors is compared in Table III, when both of them are assumed to be operated in SM-SDMA or SM/DS-CDMA systems. As shown in Fig. 12, the JSM/MMSE-IIC and MP-GSM detectors achieve nearly the same BER performance, which is significantly better than that of the MMSE-MUD. In terms of the complexity, when operated in the SM-SDMA scenario, JSM/MMSE-IIC has the same order of complexity as MMSEMUD, due to the channel-relied matrix inversion. If in this case the number $K$ of users dominates the complexity, the complexity of JSM/MMSE-IIC may be higher than that of MPGSM detector. However, in the SM/SDMA scenario, especially when massive MIMO is considered, we usually have $U>K$. In this case, the complexity of MP-GSM detector should be higher than that of JSM/MMSE-IIC, as $M_{1} M_{2}>>2$ is the usual case, and hence $U K M_{1} M_{2}>>2 U K=U K+U K>$ $U K+K^{2}$. By contrast, when SM/DS-CDMA is considered, as shown in Table I of Section III-C, the complexity of JSM/MMSE-IIC can be significantly reduced, thanks to the channel-independent weight matrix involved in the MMSEMUD. However, as shown in Table III, the complexity of MPGSM detector increases significantly, and may be much higher than that of JSM/MMSE-IIC, if wireless channels are highly dispersive. This is because MP-GSM detector has to calculate the PDFs of all observation nodes. When operated in SM/DSCDMA systems, the observations are no longer scalars, but $L$ length vectors, which result in that the complexity is depended on $L^{4}$. Additionally, the complexity of MP-GSM detector is the product of $L^{4} U K$ and the modulation order $M_{1} M_{2}$, which hence imposes the constraint on using high order modulation schemes.

\section{CONCLUSIONS}

By exploiting the joint advantages of SM and DS-CDMA, a SM/DS-CDMA system has been introduced for supporting high-connectivity MA communications. Correspondingly, a range of linear and nonlinear MUDs have been proposed and investigated. We have analyzed the single-user ABER bound of the SM/DS-CDMA systems employing JSD, and the approximate ABER of the SM/DS-CDMA systems employing MMSE-JSD. A range of formulas have been obtained, which can be used to conveniently predict the BER performance of SM/DS-CDMA systems. Furthermore, the BER performance of SM/DS-CDMA systems with various detection schemes has been investigated and compared.

From our studies and performance results, we observe that both frequency-selective fading and receive antennas may be equivalently used for different purposes, such as, to provide diversity for improving BER performance, to enhance the effectiveness of some detectors, etc. For linear MUDs, frequency-selective fading cannot be exploited for MUI mitigation. In this case, a larger number of resolvable paths results in severe MUI, yielding performance degradation. By contrast, with the aid of our proposed reliability measurement schemes, the MMSE-IIC based detectors are capable of exploiting the frequency-selective fading for MUI suppression, making these detectors significantly outperform the linear MUDs, but at the cost of slightly increased detection complexity and delay. Furthermore, both the MMSE-IIC relied detectors are able to effectively suppress MUI, even when the SM/DS-CDMA 
TABLE II

COMPARISON BETWEEN SM/DS-CDMA AND SM-SDMA EMPLOYING JML-MUD FOR GIVEN $N U$.

\begin{tabular}{ccc}
\hline & SM/DS-CDMA & SM-SDMA \\
\hline Number of receive antennas required & Small & Big \\
Bandwidth required & High & Low \\
Sensitivity to variant $K$ & Low & High \\
Sensitivity to channel estimation error & Low & High \\
Error performance (big $N U$ ) & Similar & Similar \\
Error performance at high SNR $($ small $N U$ ) & Slightly worse & Slightly better \\
\hline
\end{tabular}

TABLE III

COMPLEXITY COMPARISON BETWEEN JSM/MMSE-IIC AND MP-GSM.

\begin{tabular}{ccc}
\hline & JSM/MMSE-IIC & MP-GSM \\
\hline SM-SDMA & $\mathcal{O}\left(K U+K^{2}\right)$ & $\mathcal{O}\left(U K M_{1} M_{2}\right)$ \\
SM/DS-CDMA & $\mathcal{O}\left(K N U L+K(K+1) L^{3}\right)$ & $\mathcal{O}\left(L^{4} U K M_{1} M_{2}\right)$ \\
\hline
\end{tabular}

systems are full-load or even overload. In particular, when the number of receive antennas is sufficiently large, the BER performance achieved by the JSM/MMSE-IIC is close to the single user bound, even when the SM/DS-CDMA systems are overload. This implies that the JSM/MMSE-IIC is capable of achieving near-optimum BER performance, while requiring the implementation complexity significantly lower than that of the optimum JML-MUD.

\section{REFERENCES}

[1] R. Mesleh, H. Haas, C. W. Ahn, and S. Yun, "Spatial modulation - a new low-complexity spectral efficiency enhancing technique," in Proc. Chinacom'2006, Oct. 2006, pp. 1-5.

[2] R. Y. Mesleh, et.al, "Spatial modulation," IEEE Trans. on Veh. Tech., vol. 57, no. 4, pp. 2228 - 2241, July 2008.

[3] J. Jeganathan, A. Ghrayeb, L. Szczecinski, and A. Ceron, "Space shift keying modulation for MIMO channels," IEEE Trans. on Wireless Comm., vol. 8, no. 7, pp. 3692 - 3703, July 2009.

[4] M. Renzo, et.al, "Spatial modulation for generalized MIMO: Challenges, opportunities, and implementation," Proc. of the IEEE, vol. 102, no. 1, pp. 56 - 103, Jan. 2014.

[5] P. Yang, et.al, "Single-carrier SM-MIMO: A promising design for broadband large-scale antenna systems," IEEE Comm. Surveys \& Tutorials, vol. 18 , no. 3 , pp. 1687-1716, thirdquarter 2016

[6] I. A. Hemadeh, M. El-Hajjar, and L. Hanzo, "Hierarchical multifunctional layered spatial modulation," IEEE Access, vol. 6, pp. 94929533, 2018.

[7] X. Wu, M. Di Renzo, and H. Haas, "Adaptive selection of antennas for optimum transmission in spatial modulation," IEEE Trans. on Wireless Comm., vol. 14, no. 7, pp. 3630-3641, July 2015

[8] X. Zhou, L. Yang, C.-X. Wang, and D. Yuan, "SCM-SM: Superposition coded modulation-aided spatial modulation with a low-complexity detector," IEEE Trans. on Veh. Tech., vol. 63, no. 5, pp. 2488-2493, Jun 2014.

[9] P. Yang, Y. Xiao, Y. Yu, and S. Li, "Adaptive spatial modulation for wireless MIMO transmission systems," IEEE Comm. Letters, vol. 15, no. 6, pp. 602-604, June 2011.

[10] C. Liu, L. L. Yang, and W. Wang, "Transmitter-precoding-aided spatial modulation achieving both transmit and receive diversity," IEEE Trans. on Veh. Tech., vol. 67, no. 2, pp. 1375-1388, Feb. 2018.

[11] C. Xu, S. Sugiura, S. Ng, and L. Hanzo, "Reduced-complexity noncoherently detected differential space-time shift keying," IEEE Tran. on Sig. Proc., vol. 18, no. 3, pp. 153 - 156, Mar. 2011.

[12] M. D. Renzo and H. Haas, "A general framework for performance analysis of space shift keying (SSK) modulation for MISO correlated Nakagami-m fading channels," IEEE Trans. on Comm., vol. 58, no. 9, pp. 2590 - 2603, Sept. 2010.

[13] M. Di Renzo and H. Haas, "Bit error probability of space-shift keying MIMO over multiple-access independent fading channels," IEEE Trans. on Veh. Tech., vol. 60, no. 8, pp. 3694-3711, Oct. 2011.

[14] M. D. Renzo and H. Haas, "Bit error probability of SM-MIMO over generalized fading channels," IEEE Trans. on Veh. Tech., vol. 61, no. 3, pp. 1124-1144, Mar. 2012.

[15] M. Maleki, H. Bahrami, A. Alizadeh, and N. Tran "On the performance of spatial modulation: Optimal constellation breakdown," IEEE Trans. on Comm., vol. 62, no. 1, pp. 144-157, Jan. 2014.
[16] Z. An, J. Wang, J. Wang, S. Huang, and J. Song, "Mutual information analysis on spatial modulation multiple antenna system," IEEE Trans. on Comm., vol. 63, no. 3, pp. 826-843, Mar. 2015.

[17] B. Jiao, M. Wen, M. Ma, and H. Poor, "Spatial modulated full duplex,' IEEE Wireless Comm. Letters, vol. 3, no. 6, pp. 641-644, Dec. 2014.

[18] C. Liu, L. L. Yang, and W. Wang, "Secure spatial modulation with a full-duplex receiver," IEEE Wireless Comm. Letters, vol. 6, no. 6, pp. 838-841, Dec. 2017.

[19] R. Rajashekar, M. D. Renzo, K. V. S. Hari, and L. Hanzo, "A generalized transmit and receive diversity condition for feedback-assisted MIMO systems: Theory and applications in full-duplex spatial modulation," IEEE Trans. on Sig. Proc., vol. 65, no. 24, pp. 6505-6519, Dec. 2017.

[20] A. Garcia-Rodriguez and C. Masouros, "Low-complexity compressive sensing detection for spatial modulation in large-scale multiple access channels," IEEE Trans. on Comm., vol. 63, no. 7, pp. 2565-2579, July 2015.

[21] X. Wu, H. Claussen, M. Di Renzo, and H. Haas, "Channel estimation for spatial modulation," IEEE Trans. on Comm., vol. 62, no. 12, pp. 4362-4372, Dec. 2014.

[22] M. Di Renzo, D. De Leonardis, F. Graziosi and H. Haas, "Space shift keying (SSK-) MIMO with practical channel estimates," IEEE Trans. on Comm., vol. 60, no. 4, pp. 998-1012, Apr. 2012.

[23] L. Wang, S. Bashar, Y. Wei, and R. Li, "Secrecy enhancement analysis against unknown eavesdropping in spatial modulation," IEEE Comm. Letters, vol. 19, no. 8, pp. 1351-1354, Aug. 2015.

[24] F. Wu, R. Zhang, L.-L. Yang, and W. Wang, "Transmitter precodingaided spatial modulation for secrecy communications," IEEE Trans. on Veh. Tech., vol. 65, no. 1, pp. 467-471, Jan. 2016.

[25] Y. Chen, L. Wang, Z. Zhao, M. Ma, and B. Jiao, "Secure multiuser MIMO downlink transmission via precoding-aided spatial modulation," IEEE Comm. Letters, vol. 20, no. 6, pp. 1116-1119, June 2016.

[26] S. Wang, Y. Li, and J. Wang, "Multiuser detection in massive spatial modulation MIMO with low-resolution ADCs," IEEE Trans. on Wireless Comm., vol. 14, no. 4, pp. 2156-2168, Apr. 2015.

[27] S. Wang, Y. Li, M. Zhao, and J. Wang, "Energy-efficient and lowcomplexity uplink transceiver for massive spatial modulation MIMO," IEEE Trans. on Veh. Tech., vol. 64, no. 10, pp. 4617-4632, Oct. 2015.

[28] L. He, J. Wang, and J. Song, "Multiuser detection for FEC-coded massive spatial modulation MIMO: An iterative interference rejection approach," IEEE Trans. on Veh. Tech., vol. 66, no. 10, pp. 9567-9571, Oct. 2017.

[29] J. S. Kim, S. Lee, and M. Y. Chung, "Efficient random-access scheme for massive connectivity in 3GPP low-cost machine-type communications," IEEE Trans. on Veh. Tech., vol. 66, no. 7, pp. 6280-6290, July 2017.

[30] M. Renzo and H. Haas, "On the performance of SSK modulation over multiple-access Rayleigh fading channels," in IEEE GLOBECOM. IEEE, Dec. 2010.

[31] N. Serafimovski, S. Sinanović, M. D. Renzo, and H. Haas, "Multiple access spatial modulation," EURASIP J. on Wireless Comm. and Net., vol. 2012, no. 1, p. 299, Sept. 2012.

[32] L.-L. Yang, "Signal detection in antenna-hopping space-division multiple-access systems with space-shift keying modulation," IEEE Trans. on Sig. Proc., vol. 60, no. 1, pp. 351-366, Jan. 2012.

[33] T. Lakshmi Narasimhan, P. Raviteja, and A. Chockalingam, "Generalized spatial modulation in large-scale multiuser MIMO systems," IEEE Trans. on Wireless Comm., vol. 14, no. 7, pp. 3764-3779, July 2015.

[34] J. Zheng, "Low-complexity detector for spatial modulation multiple 
access channels with a large number of receive antennas," IEEE Comm. Letters, vol. 18, no. 11, pp. 2055-2058, Nov. 2014.

[35] D. SinanoviÄĞ, G. ÅăiÅąul, and B. Modlic, "Low-PAPR spatial modulation for SC-FDMA," IEEE Trans. on Veh. Tech., vol. 66, no. 1, pp. 443-454, Jan. 2017

[36] M. Fukuma and K. Ishii, "Space-time code division multiple access based on spatial modulation," in 2015 IEEE 82nd Veh. Tech. Conf. (VTC2015-Fall), Sept. 2015, pp. 1-5

[37] M. I. Kadir, et.al, "OFDMA/SC-FDMA aided space-time shift keying for dispersive multiuser scenarios," IEEE Trans. on Veh. Tech., vol. 62, no. 1, pp. 408-414, Jan. 2013.

[38] L.-L. Yang, Multicarrier Communications. Chichester, United Kingdom: John Wiley, 2009.

[39] Q. Wang, R. Zhang, L. Yang, and L. Hanzo, "Non-orthogonal multiple access: A unified perspective," IEEE Wireless Comm., vol. 25, no. 2, pp. 10-16, Apr. 2018.

[40] R. Hoshyar, F. Wathan, and R. Tafazolli, "Novel low-density signature for synchronous CDMA systems over AWGN channel," IEEE Trans. on Sig. Proc., vol. 56, no. 4, pp. 1616-1626, Apr. 2008.

[41] Y. Liu, L. L. Yang, and L. Hanzo, "Spatial modulation aided sparse code-division multiple access," IEEE Trans. on Wireless Comm. vol. 17, no. 3, pp. 1474-1487, Mar. 2018.

[42] Y. Du, et.al, "Efficient multi-user detection for uplink grantfree NOMA: Prior-information aided adaptive compressive sensing perspective," IEEE J. on Selected Areas in Comm., vol. 35, no. 12, pp. 2812-2828, Dec. 2017.

[43] S. Yang, et.al, "Uplink multiple access schemes for 5G: A survey," ZTE Comm., vol. S1, no. 57, pp. 31 - 40, 2017.

[44] A. Mantravadi, V. V. Veeravalli, and H. Viswanathan, "Spectral efficiency of MIMO multiaccess systems with single-user decoding," IEEE J. on Selected Areas in Comm., vol. 21, no. 3, pp. 382-394, Apr. 2003.

[45] D. Garg and F. Adachi, "Packet access using DS-CDMA with frequency-domain equalization," IEEE J. on Selected Areas in Comm., vol. 24, no. 1, pp. 161-170, Jan. 2006

[46] A. M. Tonello and R. Rinaldo, "A time-frequency domain approach to synchronization, channel estimation, and detection for DS-CDMA impulse-radio systems," IEEE Trans. on Wireless Comm., vol. 4, no. 6, pp. 3018-3030, Nov. 2005.

[47] R. Rajashekar, K. V. S. Hari and L. Hanzo, "Spatial Modulation Aided Zero-Padded Single Carrier Transmission for Dispersive Channels," IEEE Trans. on Comm., vol. 61, no. 6, pp. 2318-2329, Jun. 2013.

[48] D. Falconer, S. L. Ariyavisitakul, A. B. Seeyar and B. Eidson, "Frequency Domain Equalization for Single-Carrier Broadband Wireless Systems," IEEE Comm. Mag., vol. 40, no. 4, pp. 58-66, Apr. 2002.

[49] L.-L. Yang, "MIMO assisted space-code-division multiple-access: Linear detectors and performance over multipath fading channels," IEEE J. on Selected Areas in Comm., vol. 24, no. 1, pp. 121 - 131 Jan. 2006.

[50] P. Pan, Y. Zhang, Y. Sun, and L. L. Yang, "On the asymptotic spectral efficiency of uplink MIMO-CDMA systems over Rayleigh fading channels with arbitrary spatial correlation," IEEE Trans. on Veh. Tech. vol. 62, no. 2, pp. 679-691, Feb. 2013

[51] S. Verdu, Multiuser Detection. Cambridge University Press, 1998.

[52] H. L. V. Trees, Optimum Array Processing. Wiley Interscience, 2002.

[53] H. V. Poor and S. Verdu, "Probability of error in MMSE multiuse detection," IEEE Trans. on Inf. Theory, vol. 43, no. 3, pp. 858-871, May 1997.

[54] J. G. Proakis, Digital Communications, 5th ed. McGraw Hill, 2007.

[55] L.-L. Yang, "Receiver multiuser diversity aided multi-stage minimum mean-square error detection for heavily loaded DS-CDMA and SDMA systems," IEEE Trans. on Comm., vol. 58, no. 12, pp. 3397 - 3404 , Dec. 2010.

[56] J. Benesty, Y. Huang, and J. Chen, "A fast recursive algorithm for optimum sequential signal detection in a BLAST system," IEEE Trans. on Sig. Proc., vol. 51, no. 7, pp. 1722 - 1730, July 2003.

[57] M. K. Simon and M.-S. Alouini, Digital Communication over Fading Channels, 2nd ed. New York: John Wiley \& Sons, 2005.

[58] V. A. Aalo, "Performance of maximum-ratio diversity systems in a correlated Nakagami-fading environment," IEEE Trans. on Comm., vol. 43, no. 8, pp. 2360-2369, Aug. 1995 .

[59] P. R. Krishnaiah and M. M. Rao, "Remarks on a multivariate Gamm distribution," America Mathematics Monthly, vol. 68, no. 4, pp. 342 346, Apr. 1961.

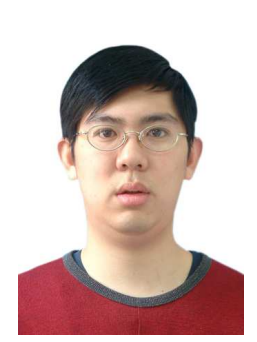

Peng Pang (M'11) received his B.Eng. and Ph.D. degrees in electronic engineering from Beihang University, Beijing, China, in 2005 and 2011, respectively. From December 2007 to December 2008, he was a Visiting Ph.D. Student to the School of Electronics and Computer Science, University of Southampton, Southampton, U.K. He is currently an associate professor in the School of Communication Engineering, Hangzhou Dianzi University, Hangzhou, China. From November 2017 to November 2018, he was a visiting scholar in the School of Electronics and Computer Science, University of Southampton, Southampton, U.K. His research interests include MIMO systems, multiuser detection, ultradense networks and performance analysis of various wireless systems.

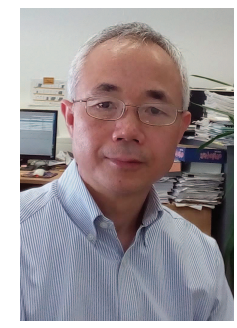

Lie-Liang Yang (M'98, SM'02, F'16) received his BEng degree in communications engineering from Shanghai TieDao University, Shanghai, China in 1988, and his MEng and PhD degrees in communications and electronics from Northern (Beijing) Jiaotong University, Beijing, China in 1991 and 1997, respectively. From June 1997 to December 1997 he was a visiting scientist to the Institute of Radio Engineering and Electronics, Academy of Sciences of the Czech Republic. Since December 1997, he has been with the University of Southampton, United Kingdom, where he is the professor of Wireless Communications in the School of Electronics and Computer Science. He has research interest in wireless communications, wireless networks and signal processing for wireless communications, as well as molecular communications and nano-networks. He has published over 370 research papers in journals and conference proceedings, authored/co-authored three books and also published several book chapters. The details about his research publications can be found at https://www.ecs.soton.ac.uk/people/llyang. He is a fellow of both the IEEE and the IET, and was a distinguished lecturer of the IEEE VTS. He served as an associate editor to the IEEE Trans. on Vehicular Technology and Journal of Communications and Networks (JCN), and is currently an associate editor to the IEEE Access and a subject editor to the Electronics Letters. 